\title{
Parametrization of Survival Measures (Part III) Clinical Evidences in Single Arm Studies with Endpoint of Overall Survival
}

\author{
Andras Szasz ${ }^{1}$, Gyula P. Szigeti², Marcell A. Szasz ${ }^{3}$ \\ ${ }^{1}$ Biotechnics Department, Faculty of Mechanical Engineering, St. Istvan University, Budaors, Hungary \\ ${ }^{2}$ Innovation Center, Semmelweis University, Budapest, Hungary \\ ${ }^{3}$ Cancer Center, Semmelweis University, Budapest, Hungary \\ Email: biotech@gek.szie.hu
}

How to cite this paper: Szasz, A., Szigeti, G.P. and Szasz, M.A. (2020) Parametrization of Survival Measures (Part III) Clinical Evidences in Single Arm Studies with Endpoint of Overall Survival. International Journal of Clinical Medicine, 11, 389-419. https://doi.org/10.4236/ijcm.2020.116034

Received: April 9, 2020

Accepted: June 6, 2020

Published: June 9, 2020

Copyright $\odot 2020$ by author(s) and Scientific Research Publishing Inc. This work is licensed under the Creative Commons Attribution International License (CC BY 4.0).

http://creativecommons.org/licenses/by/4.0/

\begin{abstract}
Many clinical trials have prospective or retrospective data-sets without comparison to the control-group formed by the same cohort as the active one. The measured single arm naturally contains the relevant information, however, in most of the cases, it is impossible to obtain it from the complex survival curve without a reference. In our previous articles [1] [2], we had shown that the self-similar Weibull distribution fits the self-organized biological mechanisms well, and so it is the best option to study the single-arm survival curves, where self-organizing process is actively present. With the Weibull decomposition of the survival curve, we can fit at least two subgroups of patients. The weighted sum of the decomposed fractions could be optimized analytically and determining the best parameters of the components and the best composition ratio of the weighted sum is also possible. In this part of our series of articles, we will show how the method works in a real clinical environment through modulated electro-hyperthermia (mEHT) as a complementary method, applied curatively when no other conventional curative therapies are available. The decomposed function of the non-responding group provides an excellent agreement with the historical controls in pancreatic cancer and non-small-cell-lung-cancer studies. In the case of glioblastoma multiform, the historical missing control from the institute where the treatment was made does not allow a comparison. We used a modified Hardin-Jones-Pauling statistical estimation and had shown in single arm clinical trials for advanced pancreas, non-small cell lung cancer and glioblastoma multiforme, that this estimation is applicable, and it is corresponding with the historical arm and with the non-responding group where this comparison was available.
\end{abstract}




\section{Keywords}

Single-Arm-Study, Weibull-Decomposition,

Hardin-Jones-Pauling-Estimation, Modulated Electro-Hyperthermia

(mEHT), Glioblastoma Multiform, Pancreatic Carcinoma, Non-Small-Cell

Lung Cancer

\section{Introduction}

Overall survival (OS) is the most reliable parameter which characterizes the efficacy of a clinical trial. Evidence-based medicine (EBM) needs statistical evidences by clinical trials [3]. The clinical evidences are categorized into phases from basic preclinical (Phase 0) to the postmarket surveillance (Phase IV) [4]. The next steps are clinical, showing safety (Phase I study), efficacy (Phase II study), and an extensive, stable applicability (Phase III study), post-sale surveillance (Phase IV study) [5]. The usual basis of statistical evaluation is the randomized separation of a well-chosen cohort to control and active arms, while being as objective and as double-blinded as possible. Extracting strong reliable evidence from single-arm survival studies is rather challenging due to the missing control in the cohort. Due to the missing control-arm, the hypothesis check is unavailable. The information about the success of the treatment is of course somehow well-embedded in the results of the active arm, but without a reference set of values, its proper selection from the data is highly difficult and, in many cases, even impossible. Double blind categorizing is when neither the patient nor the therapist has any information about the actual treatment is impossible in cases of many medical equipment approvals, because the equipment usage cannot be hidden if no other treatment is possible.

In systemic diseases like malignancies, local response is not relevant in trials, because the local responding tumor refers to the local advantage, while no data is collected for the systematic behavior of the malignancy. Late stages probably have micro or macrometastases that essentially modify the survival. Local success does not give reliable information about the survival of the patient. The decreased overall survival among others for breast carcinoma [6] [7], for non-small-cell lung cancer [8] [9], for uterine cervix [10] [11], and even for the easily "heatable" surface tumors [12] were measured together with anyway significant local response (shrinking) of the tumor. Consequently, relevant information about the success of the treatment can only be obtained, if the endpoint is OS.

The effect of active therapy that changes the patient's survival is the hidden information in the measured OS. Looking for the embedded data is hard, and the success is doubtful when only a standard data-mining is used. The actually unavailable comparison to reference could lead to misinterpretations in a single-arm study [13]. Furthermore, the enormously massive bio-variability of the 
participating individuals creates a stumbling-block for objective evaluation even in a well-chosen cohort; and covers the useful data. The life conditions (lifestyle, diet, social position, etc.) of the studied individuals are also very different; these may modify the results [14], which gets even worse when the patient uses additional supportive therapies like "home medicine", that can be picked up easily from the widely available uncontrolled internet sources.

Naturally, when the well-controlled single arm study offers obviously much better results than expected from the historical data, we tend to regard it as a breakthrough, however significant heterogeneity is observed in these comparisons [15]. When the survival is not obviously much better than the historical data, the evaluation of a single arm study is complicated and in most of the cases impossible.

A commonly applied possibility to evaluate single-arm therapy information is when researchers use a historical control from the same clinic/hospital, choose retrospectively the same conditions of the cohort-selection. The evidence of the retrospective data-collection from historical archives is, of course, weaker than the randomization. The propensity scores method offers an increased reliability of the obtained results [16] [17] by adding a database construction of the control arm to single-arm results [18]. Data mining in large and representative databases selects a comparative group of patients, with relevant and characteristic properties of the disease and the conditions of the patients, supposing that these (directly independent parameters from the actual therapy) do not change during the complete curative or palliative process. The method can be verified statistically if the confounding variables are chosen well [19]. Advanced cancerous cases limit the applicability of the propensity scores method, because the patients might have had a large variety of previously failed treatments and could develop various metastatic lesions.

For improving the statistical relevance, another method has been developed: the sequential trial [20]. It is a method during which we continue the study until the number of the patients reaches a level where it can be regarded as statistically significant. In such sequenced study, cumulative data is analyzed interim after the treatment of the chosen group of patients and a decision is made to continue or stop the given treatment at every step [21]. The sequenced trial is commonly applied in small studies [22], as a tool for evaluating the interim data for statistically significant values [23] [24] [25]. It is a useful tool for studies of advanced cases when other ways do not exist [8] [26]. This way, multiple survival endpoints could be evaluated [27]. Just like the propensity score method, the sequential method also has complications, for example when the patients are in late metastatic stages with multiple pretreatments and possible comorbidities (like organ failure or unsatisfactory laboratory results).

Oncological hyperthermia is one of the therapies that cannot be blinded, due to its machinery application, the sham treatment is usually well-sensed by the patient, so it is not possible to be blinded. Moreover, the medical staff who takes care of the patient, must also know that the treatment is a sham or not for safety 
issues. These conditions challenge the evidence, affect the reliability of these trials and make them less comparable to the evidences of conventional applications.

The chosen end-points of oncological local/regional hyperthermia clinical studies are often connected to local responses (local remission rate, local remission free survival, local progression-free survival). This choice is a logical consequence of the local treatments-however, the problem of malignancy is far beyond the local response. Malignant diseases have the possibility of forming micro- and macro-metastases by systemic dissemination far from the original tumor. The development of metastases is more life-threatening than local tumor development [28], and the invisible micro-metastases worsen the life-prognosis further [29] significantly. Unfortunately, there are multiple studies with effective and significant local control, but at the same time a decreased OS is shown in well-conducted studies among others for breast carcinoma [6] [7], for non-small-cell lung cancer [8] [9], for uterine cervix [10] [11], and even for the easily heatable surface tumors [12]. An important fact is that the inclusion criteria was "locally advanced", so no metastases were observed at inclusion. This raises doubts [30], that could block the application of hyperthermia in oncology, [31].

One of the categories of oncological hyperthermia methods is the modulated electro-hyperthermia (mEHT, trade name: oncothermia) [32]. The mEHT method is usually applied in the stages when conventional curative methods fail, and conventionally only palliation would be applicable. The method of mEHT is able to resensitize the previous refractory treatments, and usually, it is applied for late-stage patients. In most of the cases, the quality of life (QoL) is in the focus of the trials in palliative care [33]. These studies provide evidence of the palliation being mostly irrespective of the tumor-type and the selection is usually only based on the unavailability of curative approaches [34] [35].

The direct rationale of mEHT is that it attacks the malignancy in its systemic conditions, so instead of the local responses of the actual tumor, the complex issue of the overall survival with the QoL together is the usual endpoint of its studies. The basic idea behind mEHT is the selective heating of the malignant cells in a highly heterogeneous tumor. The bioelectromagnetic interactions [36] with the physiology differences of the malignant and non-malignant cells [37], allow the attack and induce the apoptosis [38], in malignant cells, while no change has been made in healthy neighboring ones. The process produces a damage-associated molecular pattern and immunogenic cell-death [39], which has a crucial role in the abscopal effect of the mEHT method [40] [41]. The immune-effect is so strong that after the treatment the re-challenging of the same tumor was unsuccessful [39]. Significant differences can be shown in a comparison of mEHT to conventional water-bath heating [42] or with other bioelectromagnetic heating methods [43].

Considering the possible controversial endpoint response-related parameters of clinical studies, the appropriately combined endpoint with QoL should be the 
overall survival (OS). The complete mEHT method was developed to solve this problem, ensuring the significant improvement of local response and the OS together [44] [45], which is viewed as the future of hyperthermia in oncology [46]. Clinical results prove that the improvement of the survival is induced by mEHT [47]. There are studies for multiple localizations, like pancreas carcinoma [48] [49] [50]; small-cell [51] and non-small-cell lung cancer [52] [53] [54]; brain gliomas [55]-[61]; uterine cervix carcinoma [62] [63]; hepatocellular carcinomas [64] [65]; sarcomas [66] [67]; biliary cancer [68], malignant ascites [69], and liver metastases [70] [71].

Our objective in this present study is to evaluate mEHT results using the single arm studies with the calculated parametric fitting to the non-parametric Kaplan-Meier (KM) estimates.

\section{Method}

First, we define the inclusion criteria for unifying the mEHT cohort. Selecting late-stage patients for mEHT in curative approach, when conventionally only palliation is available makes mEHT studies complex, and obtaining evidence difficult. The before-mentioned problems have aroused because the actual cohort contains only those late-stage patients, for whom conventional therapies are unavailable due to their refractory cases, organ failure, inadequate hematology measures, multiple relapses, or simply, there are no curative possibilities in that concrete stage of the disease. In this meaning, mEHT starts as a definitive palliation, but its intent is curative. Specialized medical facilities like hospitals, university clinics, and private services use mEHT treatment for a broadly heterogeneous group of patients who are not treatable by conventional therapies anymore. The long years of mEHT usage in oncological practice shows that the actual stage of the patient determines the time of the first, so the point of the inclusion of the patients to mEHT process is based on identical criteria: conventional curative possibilities (chemotherapy, radiotherapy, gene therapy, etc.) no longer available for this group of patients.

The blind process in a clinical study is obviously impossible in the case of mEHT. In many mEHT clinical studies, even the simple, non-blinded randomization is impossible because late-stage patients need the only applicable curative possibility, therefore, the option of exclusion from the mEHT by randomization would be unethical.

The point, when the patient leaves conventional therapy to start complementary mEHT can be regarded as the end of an independent trial. This stage is usually grouped by late palliative intent, and the aim is to provide the best supportive care. The time when previous treatments fail forms a reference point for a cohort of patients, for whom conventional curative protocols alone do not work anymore. At this point, mEHT treatment can be started and/or promoted to a complementary, but curative therapy in order to be able to use conventional approaches again. The failure of conventional therapies as the only inclusion criteria of the study unifies the mEHT cohort. In consequence, the starting point 
of $\mathrm{mEHT}$ is defined by the cohort-forming condition, therefore, the time between the diagnosis and the start of mEHT treatment $\left(T_{s}\right)$ has importance.

All the information about the efficacy of the mEHT treatment is included (but hidden) in the single arm process as well. The information describes the obtained OS, however without the reference arm, information cannot be seen. By adding quasi control-arms, the accuracy of the estimation can be improved, and the double-checking of the subgroup division becomes available, that can be compared to the historical control arm of the group with the same (but retrospective) inclusion criteria. The simplest way to create the control arm in late-stage treatments is by choosing the patients for whom conventional treatment was ineffective, or those, who were censored or deceased earlier than the end of the protocol. Note, that local response is not relevant information in trials with OS as an endpoint, because the locally responding tumor excludes the systematic behavior of the malignancy. Late-stages probably involve micro or macrometastases, which essentially modify the survival. Local success does not give reliable information about the survival of the patient.

As it was shown earlier [59] [60], the survival is expected to fulfill some universal rules originated from the self-organizing and self-similarity of the bio-structures. In consequence, the parametric Weibull function (WF) fits to the non-parametric KM plot with high accuracy. The regression curve has simple information, considering, that all the individuals in the cohort have identical fate because of the development of the malignant disease. This universality gives the possibility to extract the outliner changes from the data-coherency in the parametric curve. So, when the observed KM survival plot does not fit with appropriate accuracy by WF, the weighted sum of two or more WFs with different parameters gives a satisfactory solution [59]:

$$
\begin{gathered}
W^{(K M)}(t)=\frac{k_{1}}{N} \mathrm{e}^{-\left(\frac{t}{t_{0}^{(1)}}\right)^{n^{(1)}}}+\frac{k_{2}}{N} \mathrm{e}^{-\left(\frac{t}{t_{0}^{(2)}}\right)^{n^{(2)}}}+\cdots+\frac{k_{M}}{N} \mathrm{e}^{-\left(\frac{t}{t_{0}^{(M)}}\right)^{n^{(M)}}} \\
\text { or } W^{(K M)}(t)=\sum_{i=1}^{M} \frac{k_{i}}{N} \mathrm{e}^{-\left(\frac{t}{t_{0}^{(i)}}\right)^{n^{(i)}}} \text { and } \sum_{i=1}^{M} k_{i}=N
\end{gathered}
$$

where $M$ subgroups exist in the complete cohort of $N$ patients, and in every group, we have $k_{1}, k_{2}, \cdots, k_{M}$ patients, we introduce the WFs for every subgroup with $n^{(i)}$ and $t_{0}^{(i)}$ parameters to fit the actually measured non-parametric KM.

Other reference groups may be compared to the historical arm or make a decomposition of KM with the process of WF-fit [59]. For the usual facilities of the trial, we may group the patients roughly into two groups: responding $(r)$ and non-responding (nr). In this grouping only two subgroups of KM in (1):

$$
W^{(K M)}(t)=c_{r} \mathrm{e}^{-\left(\frac{t}{t_{0}^{(r)}}\right)^{n^{(r)}}}+c_{n r} \mathrm{e}^{-\left(\frac{t}{t_{0}^{(n r)}}\right)^{n^{(n r)}}}
$$

Due to the complete set of patients, $c_{r}+c_{n r}=1$, so (2) looks like this: 


$$
W^{(K M)}(t)=c_{r} \mathrm{e}^{-\left(\frac{t}{t_{0}^{(r)}}\right)^{n}}+\left(1-c_{r}\right) \mathrm{e}^{-\left(\frac{t}{t_{0}^{(n r)}}\right)^{n^{(n r)}}}
$$

This bi-grouping is not always possible. The measured accuracy of the obtained $W^{(K M)}(t)$ decides the necessity of further subgroups. For detailed investigation we, had chosen two single arm trials performed by mEHT: inoperable, advanced pancreas [36] and advanced non-small-cell lung cancer (NSCLC) [40], as well as for glioblastoma multiform (GBM) [42].

\section{Results}

\subsection{Inoperable Pancreas Carcinoma, a Palliative Stage with Curative Intent}

A study for the mEHT treatment of inoperable advanced pancreas carcinoma [36] involves 99 patients in the active arm from two centers (73 and 26 patients) and a historical control with 34 patients. The overall survival is shown in Figure 1.

The measured KM of historical control compared to the KM of OS of mEHT treatment is shown in Figure 2. WF can be well fitted to the historical control, but to the OS plot it is far from accurate.

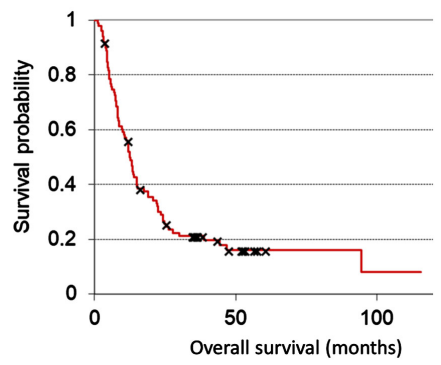

Figure 1. KM plot of OS of pancreatic cancer treated by mEHT (solid line, $n=99$, median $=12.6 \mathrm{~m}$, mean $=28.1 \mathrm{~m}$ ) with censored patients (crosses).

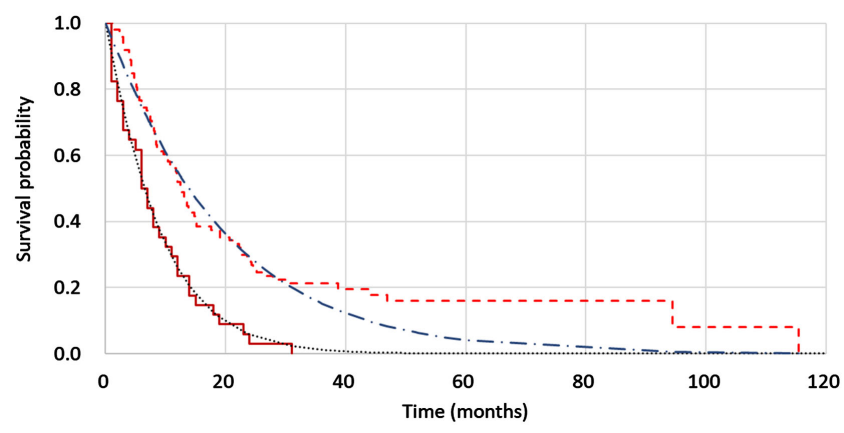

Figure 2. The KM plots of OS of pancreatic cancer treated by mEHT (dashed line) and the historical control (solid line, $n=34$, median $=6 \mathrm{~m}$, mean $=8.6 \mathrm{~m}$ ). The WF fits the parameters for control (dotted line): $n^{(c t r)}=1.08 ; t_{0}^{(c t r)}=9.17 ; S E=0.05, r^{2}=0.984$. The single WF does not fit the KM of mEHT (dash-ditted line, $n^{(O S)}=1.05 ; t_{0}^{(O S)}=19.7$; $S E=0.4, r^{2}=0.967$ ), a decomposition of responding and non-responding group of patients has to be applied. 
The WF decomposition fits significantly well to the OS (regression by minimizing the sum of deviations points by points), which is shown in Figure 3.

The comparison of the historical control and the non-responding subgroup obtained from the WF decomposition of OS in Figure 3 shows that mEHT has slightly effected even the non-responding group, but its statistical difference from the historical control is not significant ( $p=0.23$ ) (Figure 4). The information contents, that are measurable by the Shannon entropy of the Weibull probability distribution [72]; are remarkably equal in this case: $S^{(n r)}=3.15$ and

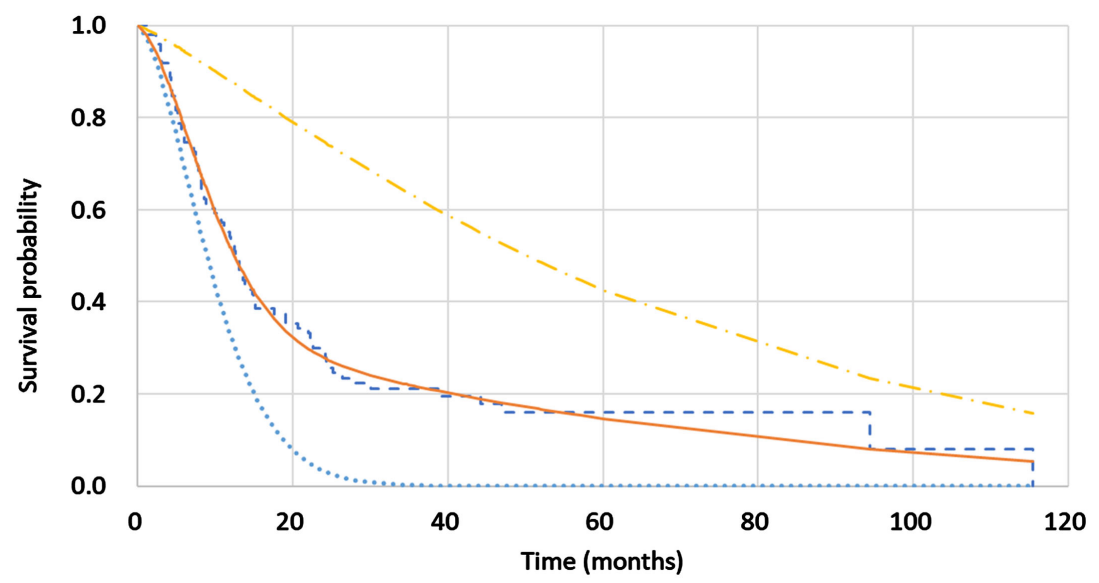

Figure 3. The Weibull decomposition fits to the OS (solid line) of pancreatic carcinoma of the advanced inoperable pancreatic cohort of patients. The Weibull parameters of "responders" (r), the dot-dashed line, and "non-responders" (nr), the dotted line are $n^{(r)}=1.18 ; t_{0}^{(r)}=68.58 ; n^{(n r)}=1.62 ; t_{0}^{(n r)}=11.19$; the percentage of the "responders" is $34.4 \%$. The sum of the two decomposed parts (dashed line) well fits to the KM. The deviation of the weighted sum of WFs from the KM estimates remains under 0.002 until $t=60$, and remains under 0.006 afterward. $S E=0.1204, r^{2}=0.995$.

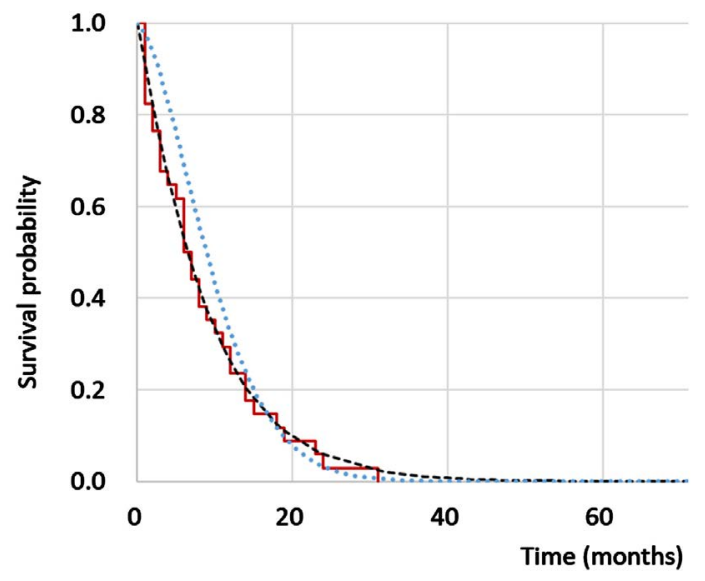

Figure 4. The non-responding component of OS of pancreatic carcinoma from Figure 3. (dotted line, $n^{(n r)}=1.62 ; t_{0}^{(n r)}=11.19 ; S^{(n r)}=3.16$ ) compared to the historical control (solid line). The parameters of the WF fit to the historical control are: $n^{(c t r)}=1.08$; $t_{0}^{(c t r)}=9.17, S^{(c t r)}=3.18$, (dashed line). No significant difference is observed $(p=0.23)$, and the Shannon entropies show the equivalence. 
$S^{(c t r)}=3.18$, which supports the statistical equivalence of the non-responding group from single non-parametric Kaplan-Meier with the historical control. This equivalence well verificates the decomposition concept by identifying the responding and non-responding patients, and so forming references to the single-arm study. This is not only a simple reference, but at the same time, it shows the percentages of the patients, whom the active treatment helps.

Despite the late stages and the conventionally palliative phase of the inoperable pancreatic patients, mEHT had shown curative features. The survival time from the first mEHT application has $6.1 \mathrm{~m}$ median (Figure 5).

Studying the KM of the time from the first mEHT treatment gives another opportunity for controlling the WF approach. Decomposing the KM by WFs, the sum properly fits the measurement (Figure 6).

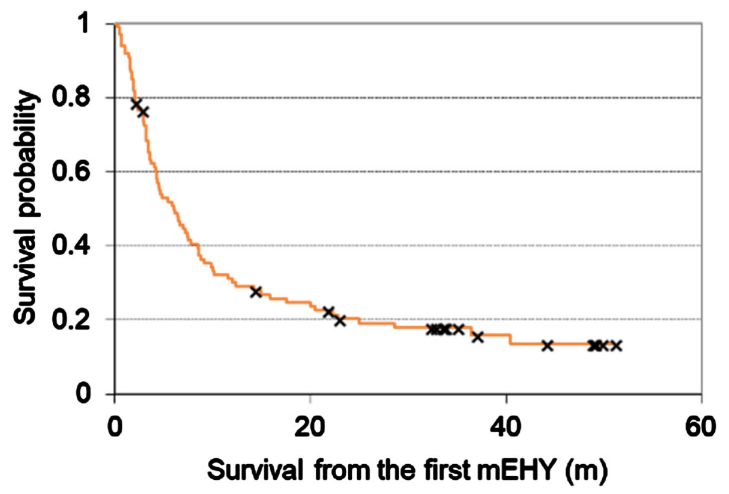

Figure 5. Survival probabilities of inoperable pancreatic carcinoma treated by mEHT ( $n$ $=99$, median $=6.1 \mathrm{~m}$, mean $=14.1 \mathrm{~m})$. The survival is measured from the first mEHT treatment, practically when the patient loses the possibility of curative intent by conventional therapies. The censored patients are denoted by a cross.

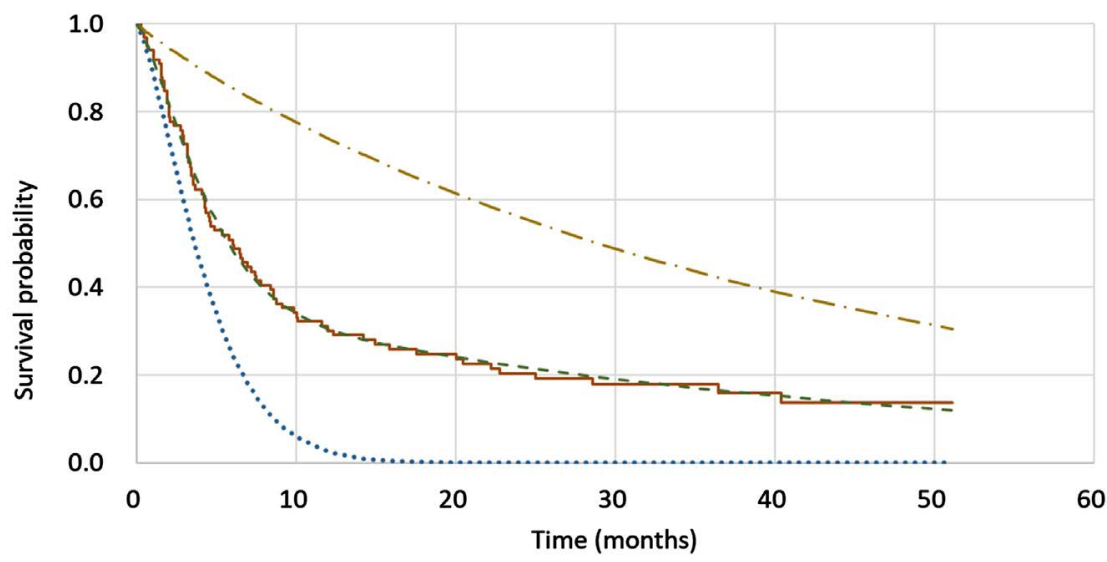

Figure 6. The KM of the survival distribution of pancreatic carcinoma by the time from the first mEHT treatment (solid line); and its best WF fit (dashed line). The accuracy of the regression is: $r^{2}=0.9966, S E=0.0367$. The WF decomposition has a responding (dot-dashed line, $n^{\left(m E H T_{-} r\right)}=0.94 ; t_{0}^{\left(m E H T_{-} r\right)}=42.67$ ) and a non-responding group (dotted line, $\left(n^{\left(m E H T_{-} n r\right)}=1.39 ; t_{0}^{\left(m E H T_{-} n r\right)}=4.74\right)$. The percentage of the responding patients is $c^{(r)}=39.2 \%$. 
The decomposition percentage (39.2\%) for the responding subgroup of the mEHT period is higher by $4.8 \%$ than the percentage of the same group in complete OS. Similarly to the results of the OS's decomposition number in Figure 3, it is over $30 \%$, support further the previously observed accuracy of the WF decomposing fit.

\subsection{Non-Small-Cell Lung Cancer (NSCLC) Palliative Phase Curative Intent}

Another clinical trial was conducted for the advanced NSCLC by mEHT treatment [40], where patients were selected based on their finished conventional therapies without curative possibilities. The study involves 258 patients from two centers (197 and 61 patients, respectively) and a historical control from another hospital including 53 patients. Figure 7 shows the overall survivals for the two centers and the completely unified cohort.

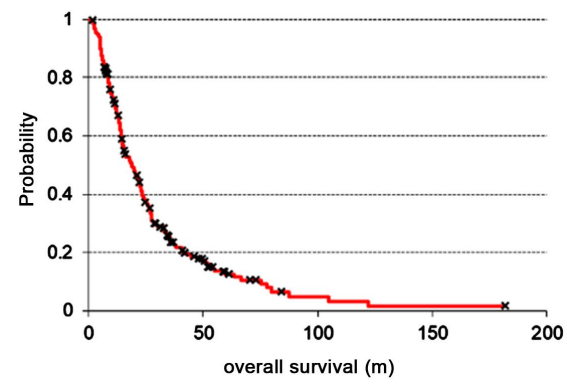

(a)

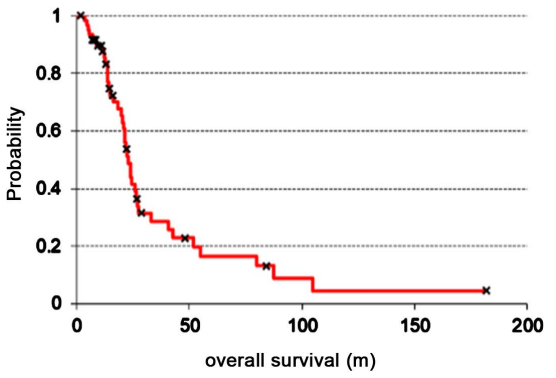

(b)

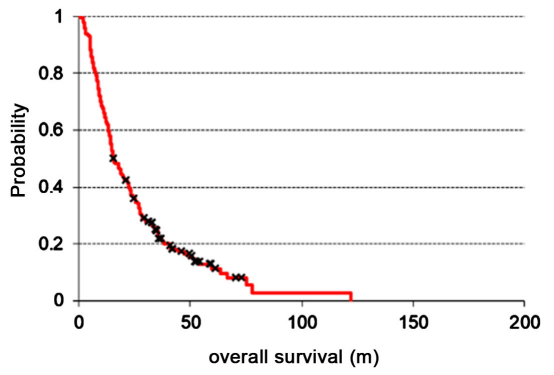

(c)

Figure 7. KM plot of OS of NSCLC treated by mEHT with censored patients (crosses). (a) all mEHT treated patients from the two centers $(n=258$, median $=18.97 \mathrm{~m}$, mean $=$ $29.59 \mathrm{~m})$, (b) center A, governmental hospital $(n=61$, median $=23.03 \mathrm{~m}$, mean $=37.88$ $\mathrm{m})$, (c) center B, private hospital $(n=197$, median $=15.8 \mathrm{~m}$, mean $=26.2 \mathrm{~m})$. 
The measured KM of the historical control (in the case of patients who were treated with only palliative treatments, due to failed curative possibilities) compared to the KM of the OS of mEHT treatment is shown in Figure 8. WF can be well fitted to the historical control, but fitting to the OS plot it is far no accurate.

The WF decomposition, a regression by minimizing the sum of deviations data by data, fits to the OS of NSCLC is shown in Figure 9 significantly well.

The comparison of the historical control and the non-responding subgroup obtained from WF decomposition of OS in Figure 8 shows that mEHT has slightly affected even the non-responding group, but its statistical difference from the historical control is not significant (Figure 10). The information coincidences well: $S^{(n r)}=3.56$ and $S^{(c t r)}=3.85$, which supports the statistical equivalence of the non-responding group from the single non-parametric Kaplan-Meier with the historical control. This equivalence verifies the decomposition concept well by identifying the responding and non-responding patients,

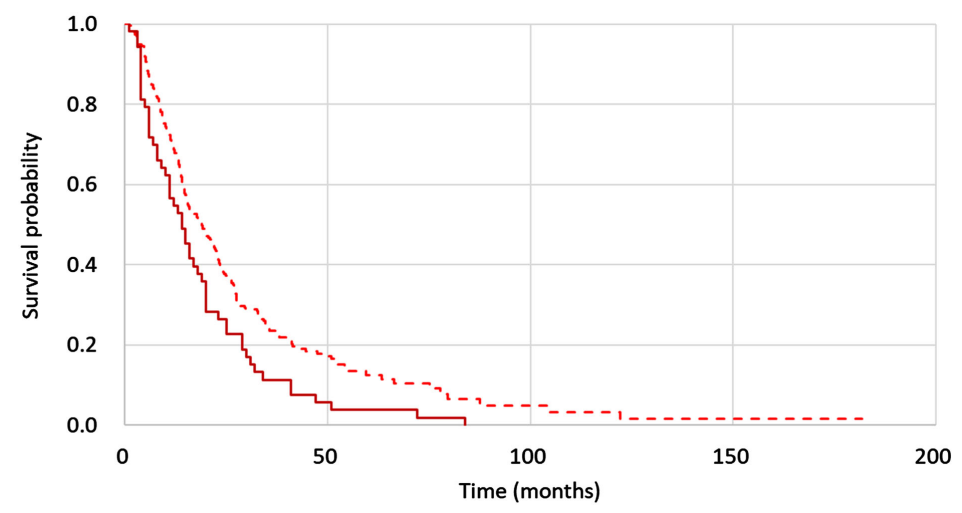

(a)

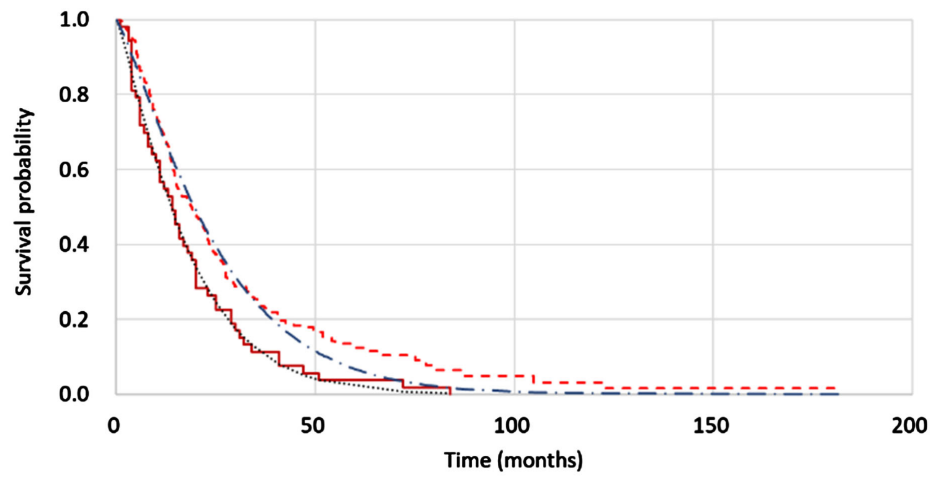

(b)

Figure 8. (a) KM plots of the OS of NSCLC treated by mEHT (dashed line, $n=258$, median $=18.97 \mathrm{~m}$, mean $=29.59 \mathrm{~m})$, and the historical control (solid line, $n=53$, median $=$ $14.0 \mathrm{~m}$, mean $=18.53 \mathrm{~m}$ ). The difference between the OS of the mEHT arm and the control arm is strongly significant $(p<0.0045)$ (b) The WF fit parameters for control (dotted line $\left.n^{(c t r)}=1.19 ; t_{0}^{(c t r)}=18.83 ; S E=0.045, r^{2}=0.992\right)$. Single WF does not fit the $\mathrm{KM}$ of mEHT (dash-dotted line, $n^{(O S)}=1.21 ; t_{0}^{(O S)}=26.56 ; S E=0.353, r^{2}=0.989$ ), the decomposition of responding and non-responding group of patients has to be applied. 


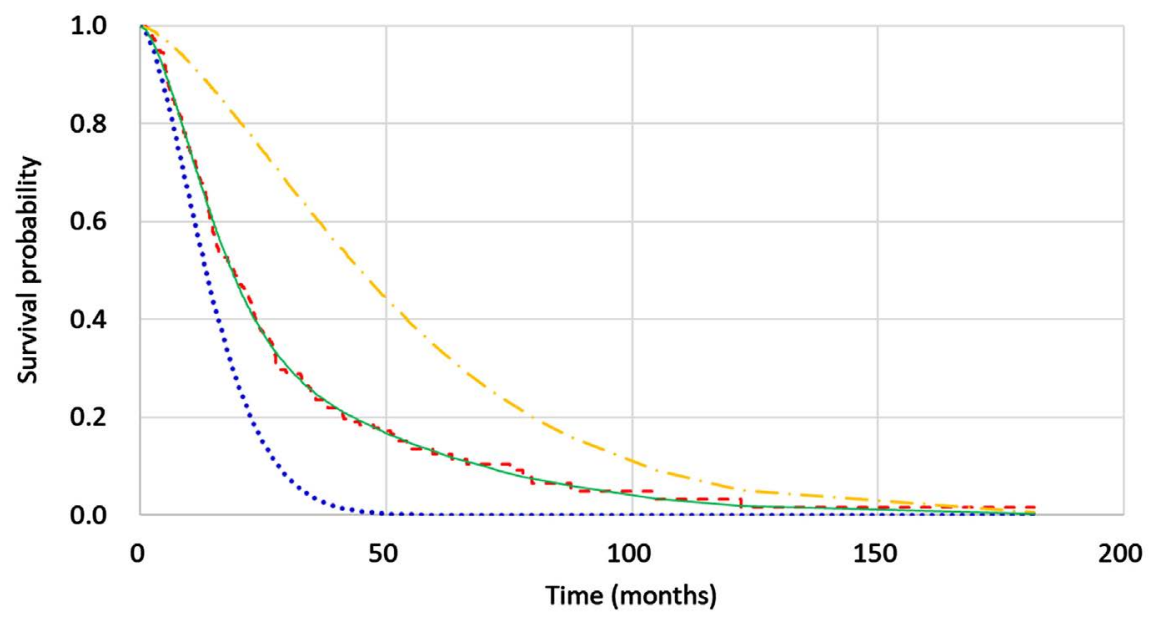

Figure 9. The Weibull decomposition fits for the OS (dashed line) of NSCLC of advanced stages of the disease. Weibull parameters of "responders" (r), dot-dashed line, and "non-responders" (nr) dotted line are $n^{(r)}=1.45 ; t_{0}^{(r)}=57.59 ; n^{(n r)}=1.61 ; t_{0}^{(n r)}=16.73$; the percentage of the "responders" is $37.4 \%$. The sum of the two decomposed parts (solid line) fits to the KM, $S E=0.062, r^{2}=0.9980$ well.

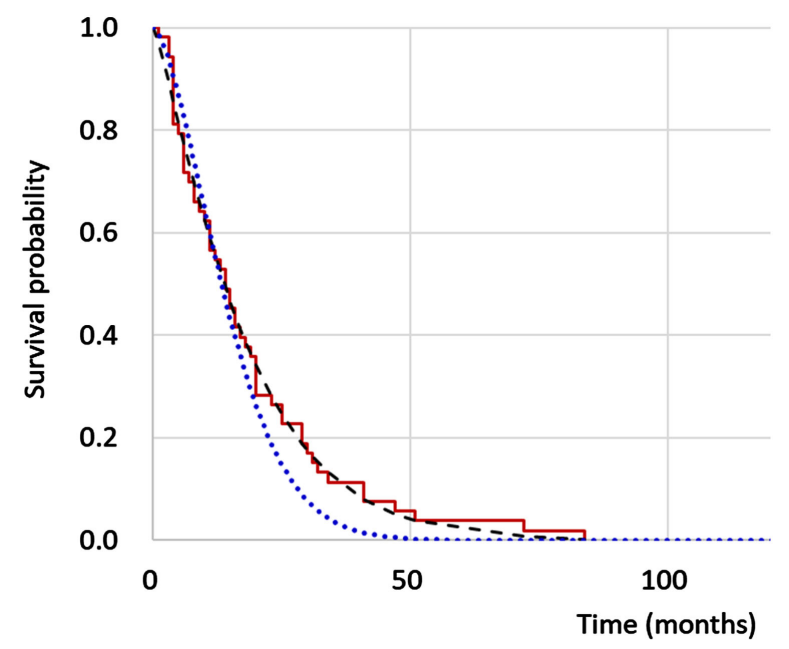

Figure 10. The non-responding component of the OS of NSCLC from Figure 8. (dotted line) compared to the historical control (solid line). The WF fit of the historical control (dashed line) has no significant difference from the non-responding group of mEHT treated patients.

and so forming references to the single-arm study. This is not only a simple reference, it shows the percentage of the patients, who the active treatment has helped as well.

The KM curve is approached well by the sum of two WF with decomposition sub-curves of responding and non-responding patients (Figure 11).

The percentage of responding patients in the decomposition of the OS of mEHT treatment time (48.1\%) is better by $10.4 \%$ compared to the OS and similarly to the result with the decomposition number of OS in Figure 9, it is over $37 \%$, supporting the previously observed accuracy of the WF decomposing fit. 


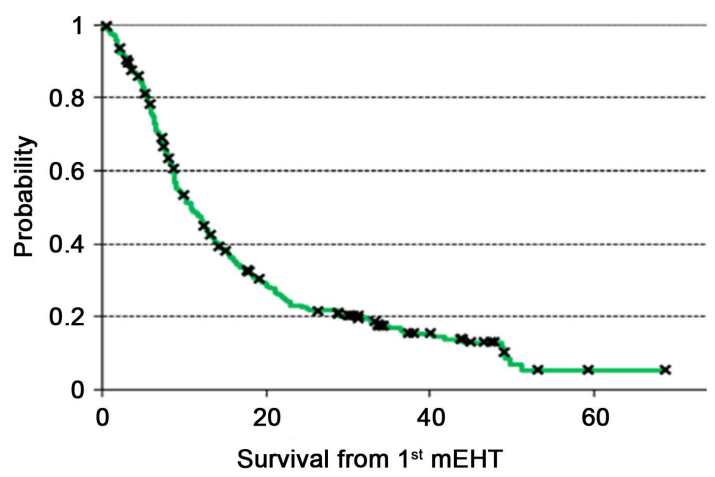

(a)

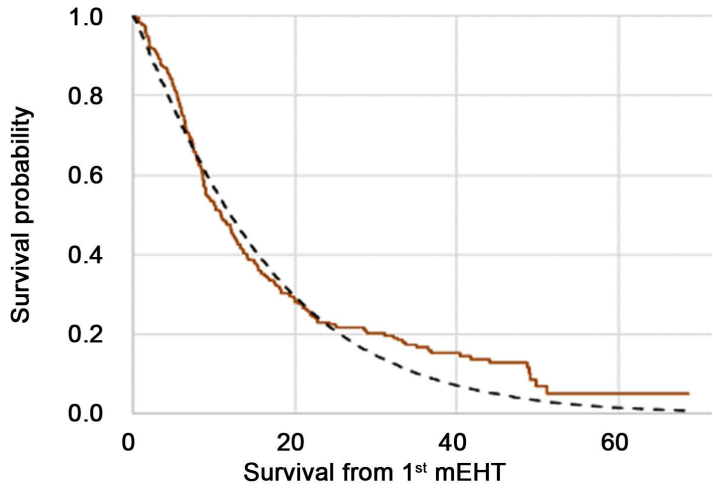

(b)

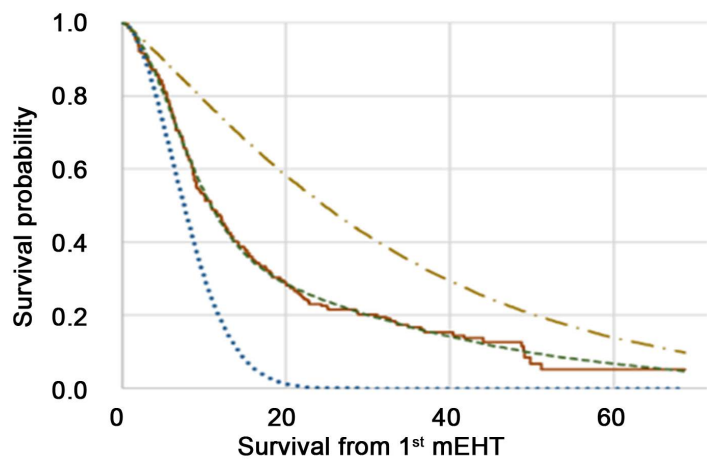

(c)

Figure 11. The elapsed time after the first mEHT treatment, when the conventional treatment has reached the palliative phase. (a) the measured KM plot from the start of the mEHT treatment periods; (b) A single WF regression (dashed line) does not fit well to the measured KM plot. (c) The WF decomposing produces perfect fit (dashed line) to the measured KM (solid line). The parameters of the responding and non-responding components: $n^{(r)}=1.18 ; t_{0}^{(r)}=33.7 ; n^{(n r)}=1.89 ; t_{0}^{(n r)}=9.2$; the percentage of the "responders" is $48.1 \%$. The sum of the two decomposed parts (solid line) well fits to the KM, $S E=0.057, r^{2}=0.998$.

\subsection{Glioblastoma Multiform}

A clinical trial for the advanced GBM was performed by mEHT treatment after the conventional therapies had no more curative possibilities [42]. The study involves 94 patients. The overall survival is shown in Figure 12. 


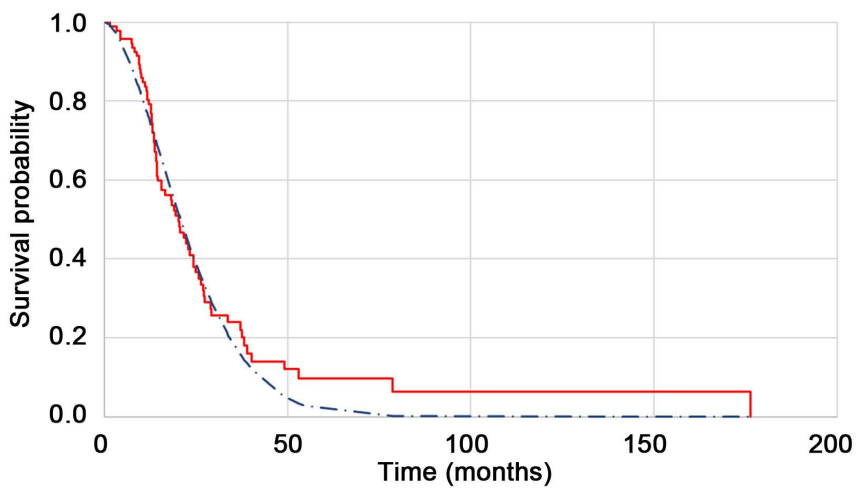

(a)

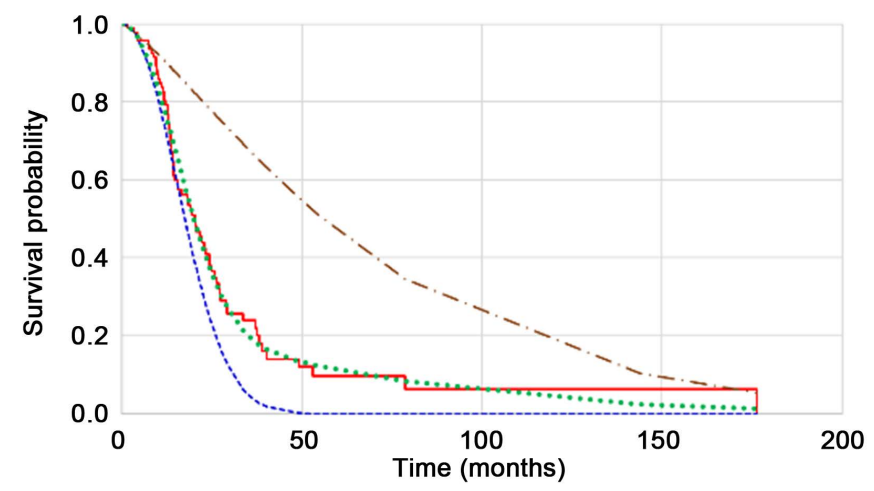

(b)

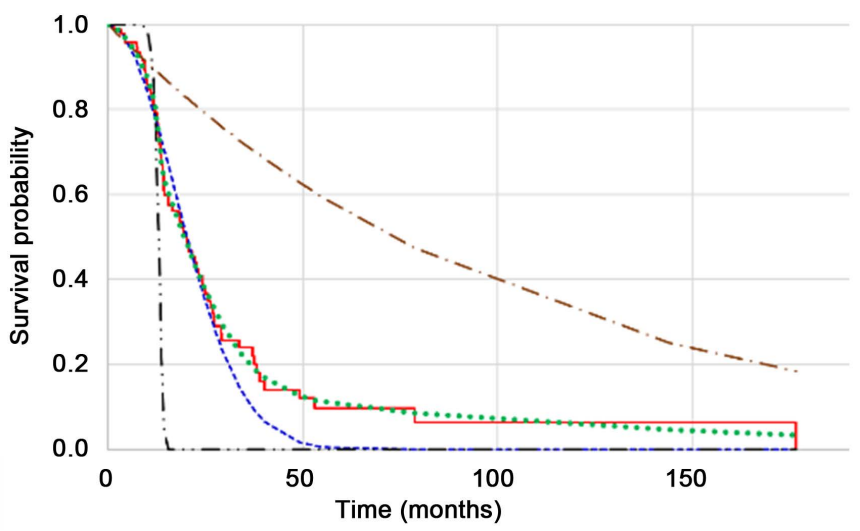

(c)

Figure 12. The overall survival including the mEHT treatment finishing, when the conventional treatment has reached the palliative phase. (a) the measured KM plot (solid line) with a single WF regression curve, (dashed-dotted line; $n^{(1)}=1.69 ; t_{0}^{(1)}=25.81$; $S E^{(1)}=0.295 ; r_{1}^{2}=0.975$ ); (b) regression with a decomposed WF. Two parts are applied, responders (dashed-dotted line, $n^{(r)}=1.25, t_{0}^{(r)}=75, c^{(r)}=25 \%$ ) and non-responders (dotted-line, $n^{(n r)}=2.11, n^{(n r)}=2.11, c^{(n r)}=75 \%$ ). The sum of the parts (dotted line; $S E=0.078, r^{2}=0.993$ ) fits with the error. (c) Regression with decomposition into three parts. Superior responders (dashed-dotted line, $n^{(s r)}=1.2, t_{0}^{(s r)}=124.6, \quad c^{(s r)}=15.6 \%$ ); responders (dashed line, $n^{(r)}=1.95, t_{0}^{(r)}=24.3, c^{(r)}=14.9 \%$ ) and non-responders (dashed-double-dotted line, $n^{(n r)}=15.6, t_{0}^{(n r)}=13.4, c^{(n r)}=69.5 \%$ ), and the sum of the parts (dotted line, $S E=0.038, r^{2}=0.997$ ), shows the best fit. 
The overall survival curve in case of GBM cannot be fitted by two regression curves (responders and non-responders). The non-responders are unfortunately large $(67.5 \%)$, but the responders' group has two subgroups, superior response (17.5\%) and response (15\%). The survival from the first mEHT treatment (finalizing the complete therapy set) shows the same behavior (Figure 13), but much fewer non-responders (14.7\%) in this part of the treatment.

It is remarkable that the non-responding subgroup in the period of mEHT treatment is only $14.7 \%$ (compared to the complete OS, where it was $67.5 \%$ ).

\section{Discussion}

By the proper decomposition of KM to WF sub-groups, we were able to unhide the well-buried information in the single-arm study, and we were able to see the percentage of responding and non-responding patients when fitting WF-curves by best regression possibilities. The pancreas and NSCLC analyses had an accurate decomposition of KM into two parts, but GBM needed three subgroups for an accurate regression. The analysis of the elapsed time until the first mEHT treatment compared to the time when the mEHT was active shows huge differences between the groups, Figure 14. While the two KM plots are well-distinguishable in the pancreas and NSCLC cases. However, the elapsed time to the first mEHT and the survival from that have similar curves in GBM plots.

The comparison of OS KM curves to the KM of mEHT involvement (Figure 15). It is obvious (that can be seen from the Figure 14 too), that the survival

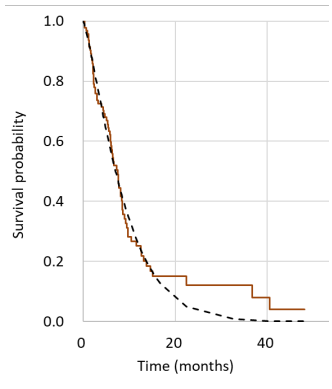

(a)

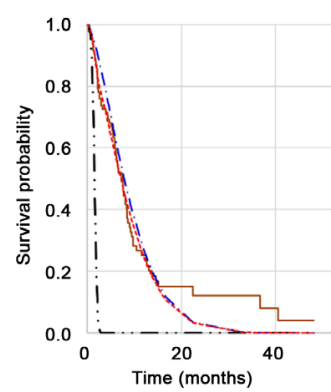

(b)

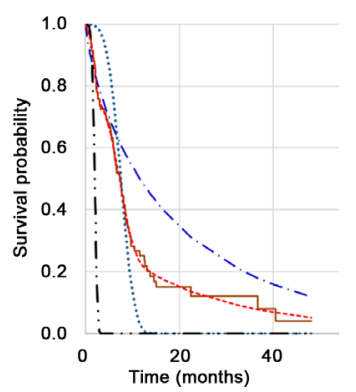

(c)

Figure 13. The survival from the start of the mEHT treatment, when the conventional treatment has reached the palliative phase. (a) the measured KM plot (solid line) with a single WF regression curve, (dashed line; $n^{(1)}=1.26 ; t_{0}^{(1)}=9.44 ; S E^{(1)}=0.175$;

$r_{1}^{2}=0.985$ ); (b) regression with a decomposed WF. Two parts are applied, responders (dashed-dotted line, $n^{(r)}=1.51, t_{0}^{(r)}=10.1, c^{(r)}=91 \%$ ) and non-responders (dasheddouble-dotted-line, $n^{(n r)}=3.85, t_{0}^{(n r)}=1.76, \quad c^{(n r)}=9 \%$ ). The sum of the parts (dashed line; $S E=0.16, r^{2}=0.987$ ) fits with the error. (c) Regression with decomposition into three parts. Superior responders (dashed-dotted line, $n^{(s r)}=0.79, t_{0}^{(s r)}=18.47$, $c^{(s r)}=43.8 \%$ ); responders (dotted line, $n^{(r)}=3.8, t_{0}^{(r)}=8.34, c^{(r)}=41.5 \%$ ) and nonresponders (dashed-double-dotted line, $n^{(n r)}=4.82, t_{0}^{(n r)}=2.17, \quad c^{(n r)}=14.7 \%$ ), and the sum of the parts (dotted line, $S E=0.034, r^{2}=0.997$ ), shows the best fit. 


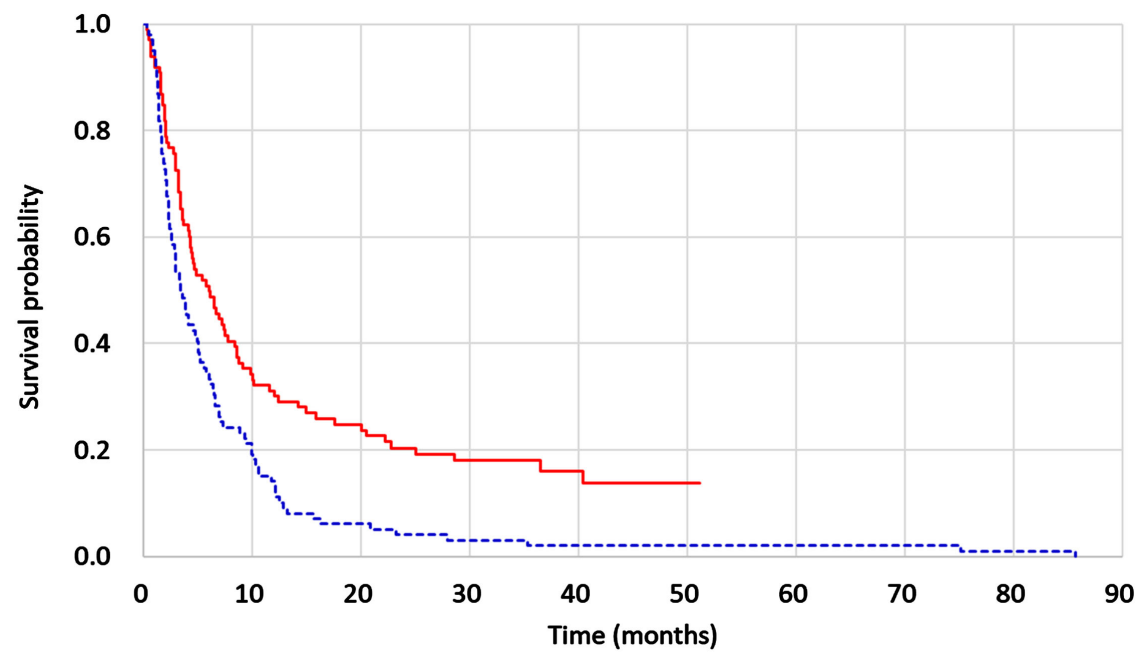

(a)

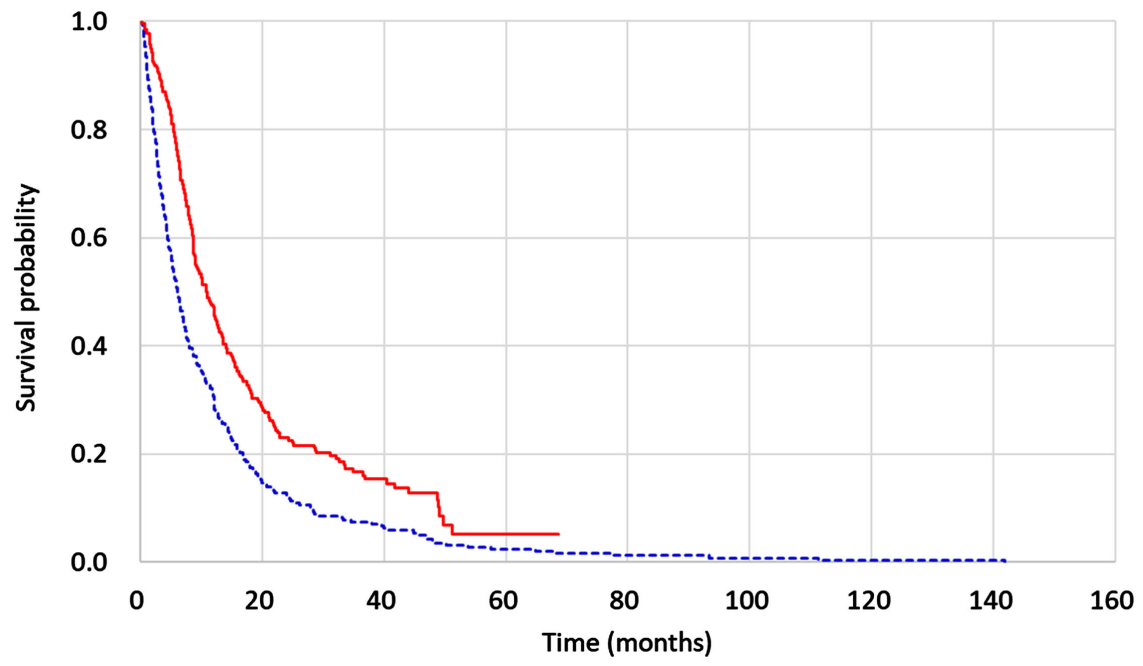

(b)

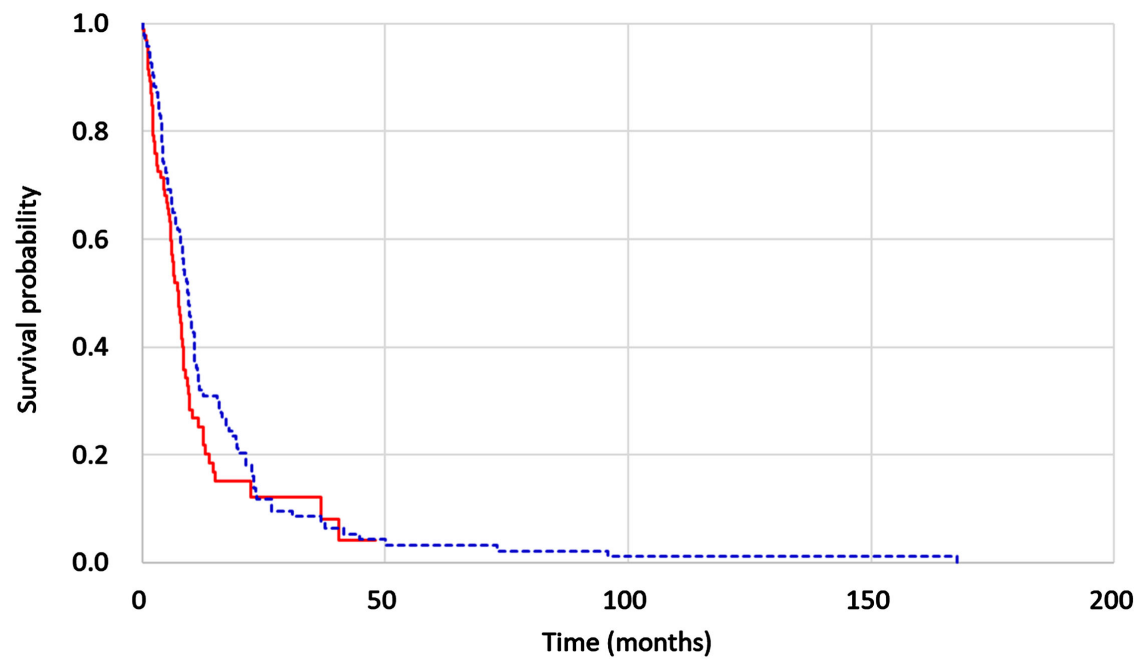

(c)

Figure 14. Comparison of the elapsed time until the mEHT process (dotted line) to the time from the first mEHT treatment (solid line), (a) pancreas; (b) NSCLC; (c) GBM. 


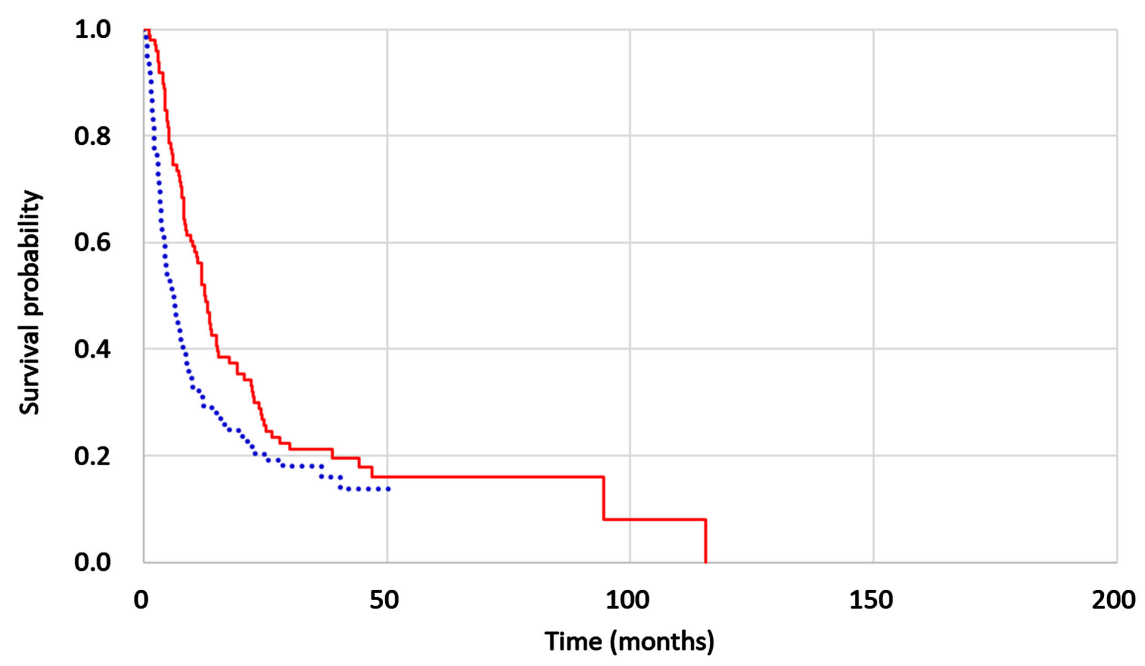

(a)

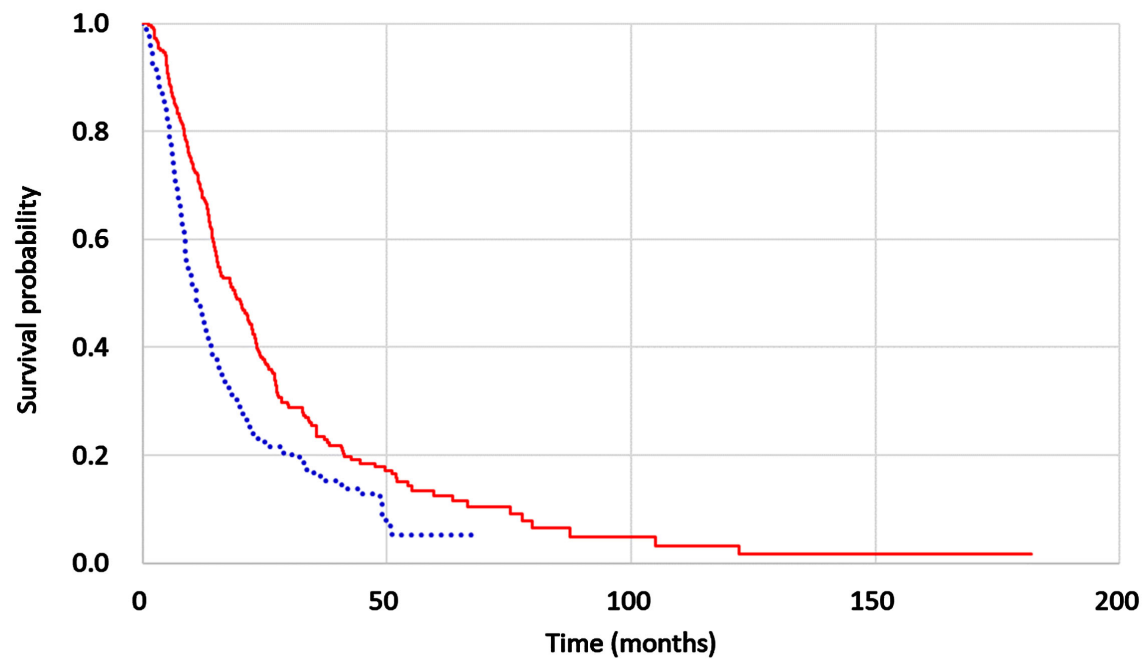

(b)

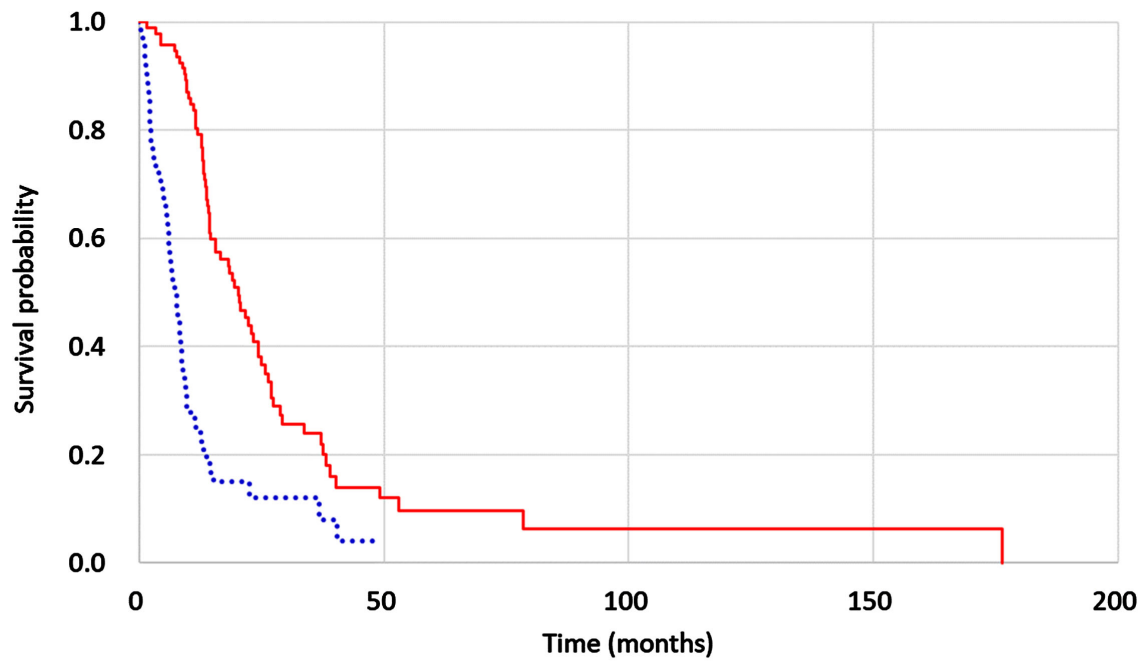

(c)

Figure 15. Comparison of the OS (solid line) and the time from the first mEHT treatment (dotted line), (a) pancreas; (b) NSCLC; (c) GBM. 
time from the start of mEHT is too short in GBM cases compared to the pancreas and NSCLC studies. The reason is probably the fact, that GBM does not create metastases, in case of relapse, multiple intracranial surgeries can be done, therefore a longer time is available for conventional treatments than in other cases.

A tool was proposed to evaluate single arm data-collection [73], focusing on the survival of censored patient's after leaving the study. Knowing the possible survival of these censored patients is crucial for the evaluation, regarding them as a control group with patients whose lifetimes were not modified by the further therapy. The proposal of this method was elaborated by Pauling and was worked out theoretically later, known as the Hardin-Jones-Pauling (HJP) biostatistical theory [74] [75]. The HJP approximation calculates the event after censoring, when the patient leaves the study before its end and his/her condition is unknown afterward. The approximation is rather simple: adding the average survival of the study to the actual time when the censoring happens:

$$
t_{H J P}=t_{\text {actual }}+\tau
$$

where $\tau$ is the mean value of the survival in the last treatment-line period. This calculation gives a quasi-reference value for all the patients comparing the expectancy of the survival time to the patients who completed the therapy. The HJP approximation can be applied self consistently as a new arm [76]. An independent, but similar concept was used recently with the mean of OS, extrapolating the long-surviving "tail" in KM curve [76]. The asymptotic properties of the estimator showed a proper coverage probability.

A method to find the reference to the single arm study could be developed by the HJP biostatistical principle. Patients, who complete a given line of treatment could be newly diagnosed in a more serious stage and could continue the treatment in a higher line. We may regard, that the finished treatment line, when no conventional curative treatment is available anymore is a clinical treatment period with the endpoint determined by the patient's need for the next line of treatment. This next line is the mEHT combined curative period. The KM plot from the first diagnosis to the first mEHT treatment refers to the probability of leaving the conventional curative protocols, due to further unavailability. In this $\mathrm{KM}$ approach, all the patients are censored at the end, leaving the conventional protocol, and starting the mEHT. The HJP approximation from the conventional treatment period is independent from the mEHT, consequently could be used as a assumption of the overall survival, and so as a control group, derived by the HJP approximation. However, this approach has a great challenge. The threshold when the curative treatment stops could create two groups of patients.

Patients whom conventional treatments could not help, and who very quickly run out of possibilities (have no effective improvement or are blocked by hematological reasons, organ failure or comorbidities), or patients, who despite having long-term benefits from conventional treatments reach the overall limit of application when the disease becomes refractory. 
Patients from both groups start the mEHT treatment with very different backgrounds and prognoses. Patients, who have been in the conventional period for a long time have already had a condition, in which the defense mechanism against the cancer was strong, while patients in the 2 nd group have had a poor defense against the malignancy. The defense mechanism covers multiple factors: genetic behavior, social situation, family environment, general condition (including immune condition), comorbidities or susceptibility to comorbidities, psychological factors (including susceptibility to depression), etc. The distribution of these factors is unknown, but due to their common occurrence in a large number of participants and the central limit theorem [77], the sample means are close to a normal distribution when the sample size is over 30. According to this, the mean of all samples is approximately equal to the mean of the population, and independent from the shapes of the population distributions.

The KM plot that fits the elapsed time between the first diagnosis and the first mEHT treatment (Figure 16), could be the basis for the approximation of the further survival of the patients after starting the mEHT.

All of those are eligible for the new line, but everybody is censored at the end of the previous treatment protocols. The KM plot's mean (average) value is $\langle$ pre_mEHT $\rangle=t_{0}^{(0)} \Gamma\left(1+1 / n^{(0)}\right) \cong 5.41$, which according to (4) is the elongation period of the expected survival time of the patients leaving the actual conventional protocol, shown in Figure 17.

In this approach, the HJP estimate is a simple time-shift of the distribution of elapsed time to the first mEHT treatment form the first diagnosis. This could be used as a control arm for the mEHT treatment, but it is not automatic. The situation is different, if the patient kept in the line of conventional therapies longer than the therapy is effective enough (for example after the second relapse). In this case, the long time, that elapsed until the first mEHT, will show a longer

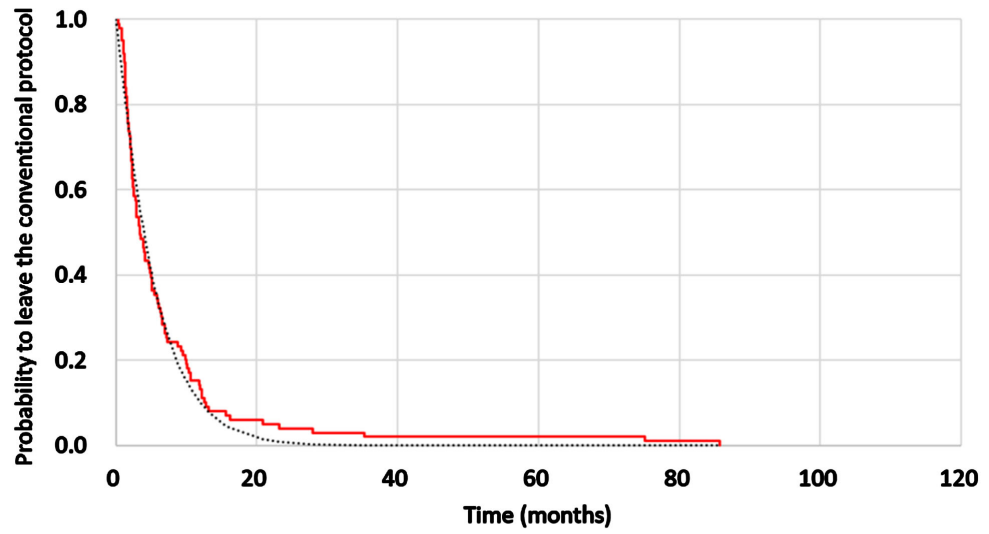

Figure 16. The time when finishing conventional lines and starting mEHT for pancreatic carcinoma from Figure 3. (solid line, $n=99$, median $=3.4 \mathrm{~m}$, mean $=7.1 \mathrm{~m}$ ). The WF fit to it (dotted line, $n^{(0)}=1.08 ; t_{0}^{(0)}=5.57, r^{2}=0.982, S E=0.294 ;$ mean $=5.41$, median $=3.97, S^{(0)}=2.68$ ). The WF fits, no significant difference is observed between the curves. 


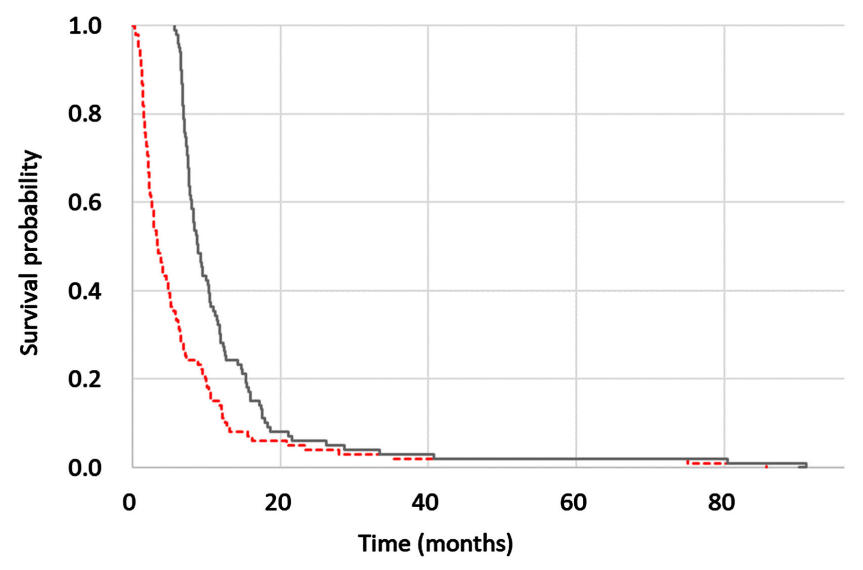

Figure 17. The HJP estimate (solid line) of pancreatic carcinoma from the survival to the first mEHT treatment. The elapsed time-distribution between the first mEHT and the first diagnosis is shown with a dashed line.

time for survival by the control arm with HJP estimates, due to the addition of the average value of the time to mEHT. To get over this complication, we can apply a normal distribution as a modifier of the simple $\tau$ average of elapsed time until the mEHT involved treatment period. So the modified KM $(\mathrm{KMm})$ of the parametric estimate which time series at (4) will be rewritten like:

$$
K M m\left(t_{H J P}\right)=\frac{K M\left(t_{H J P}\right)}{\alpha \sqrt{2 \pi \sigma^{2}}} \exp \left(-\frac{\left(K M\left(t_{e}\right)-\mu\right)^{2}}{2 \sigma^{2}}\right)
$$

where $K M\left(t_{e}\right)$ is the probability of the KM-plot of elapsed time from the first diagnosis to the first mEHT, $\mu$ is the mean and $\sigma$ is the standard deviation $\left(\sigma^{2}\right.$ is the variance) of the distribution; and $\alpha$ is a normalizing factor. The parameters are fixed by physical assumptions (Figure 18):

1) The $\mu=0$ was chosen to fix the strict monotony of the plot.

2) The $\alpha$ was chosen to have the function value 1 at the mean of the distribution.

3) For the $\sigma$ we use the percentage of the non-responding patients, as a dividing parameter for groups of patients in weak and strong condition. The group which is higher than $\sigma$, has a lower extrapolated survival. They start the mEHT earlier than the limit made by non-responders, and other patients are late starters, due to their defense systems, that can be regarded stronger.

The modified HJP (HJPm) and the elapsed time from the first diagnosis to the first mEHT show significant differences (Figure 19); the elapsed time bases the approximated expected survival well after the failure of the conventional curative therapies.

The comparison of the HJPm survival curve, the historical control and the non-responding fraction from the WF decomposition, the three control arms are practically equivalent (Figure 20).

In the case of GMB, no historical control exists, therefore we may compare the OS and the HJPm approximation. The WF decomposition, in this case, has three 


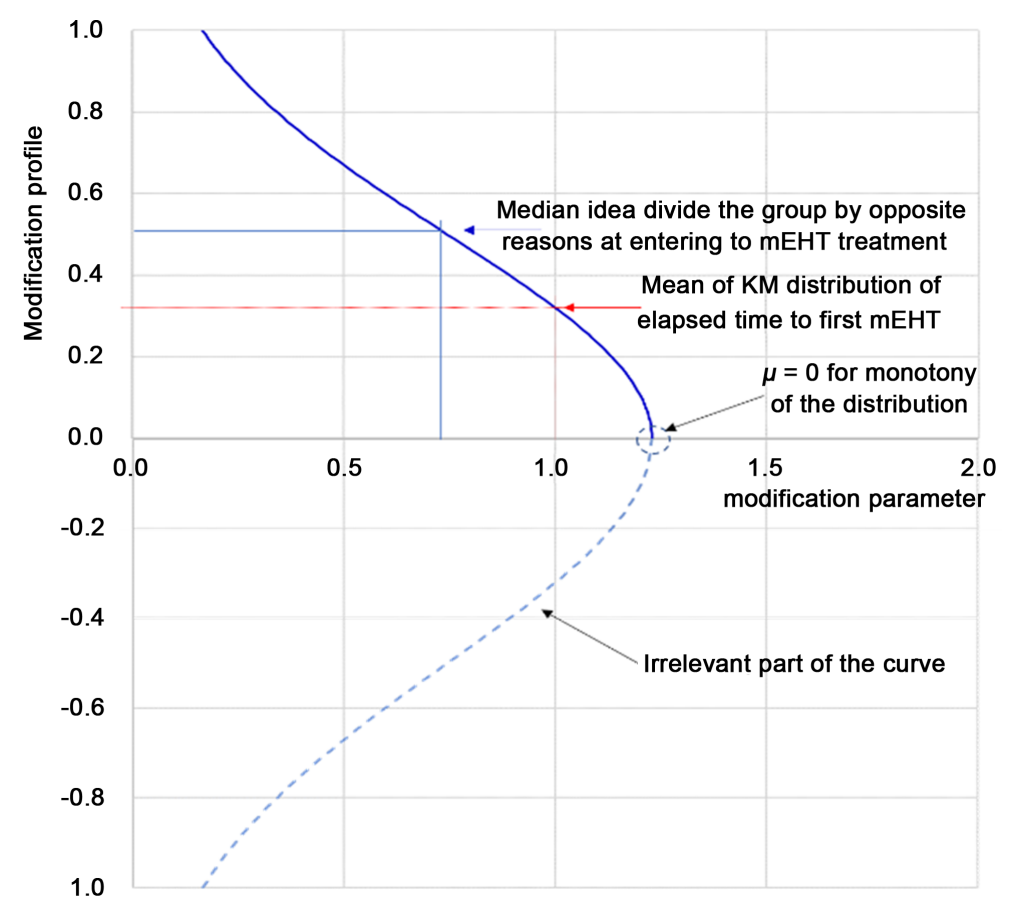

(a)

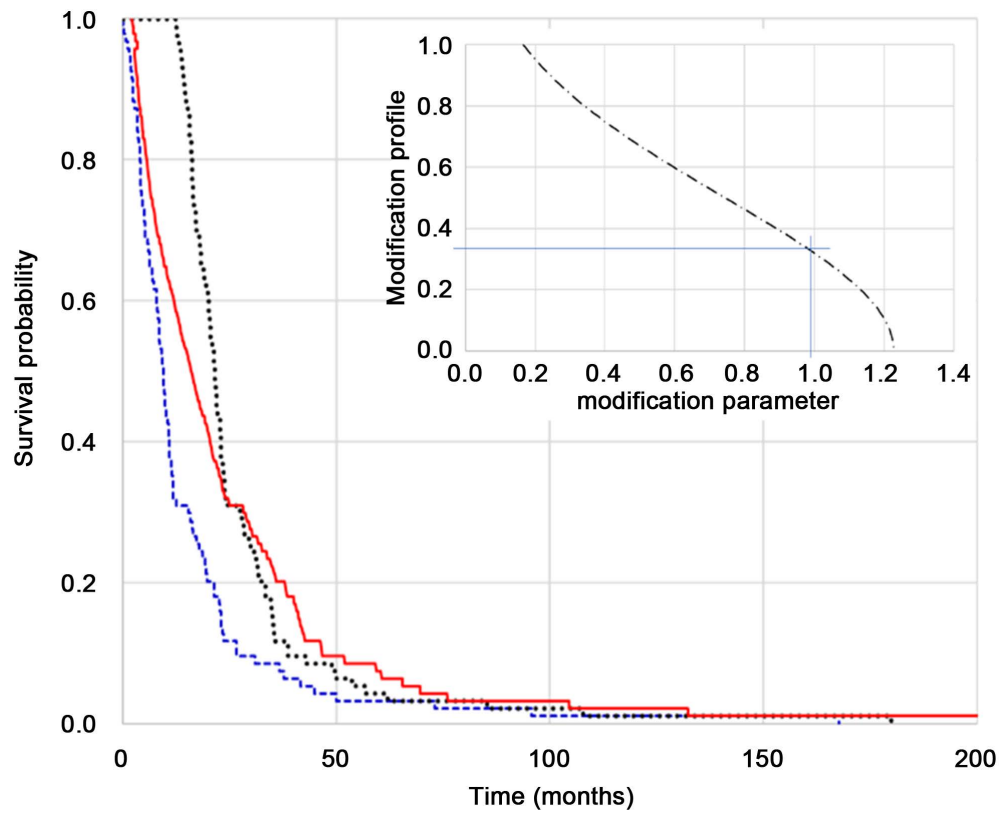

(b)

Figure 18. The HJP (HJPm) principle modified by the differences of the patients leaving the conventional curative treatment period. For simplicity, we used the median $(50 \%-$ $50 \%$ of patients in weak and strong conditions). Below the median shows patients who have left early due to insufficient improvement or personal weakness; while the patients over the median have appropriate defense and/or benefited from the treatments for a long time. (a) the multiplication function. Here only the positive (solid line) part is used, the negative (dashed line) is shown only for clarity; (b) The HJPm estimation based on the elapsed time until the first mEHT treatment (for GBM) have shown the elapsed time (dashed line) and its HJP approximation, a shift by mean (dotted line) and the HJPm curve (solid line). The modification function (normal distribution) is shown in the insert. 


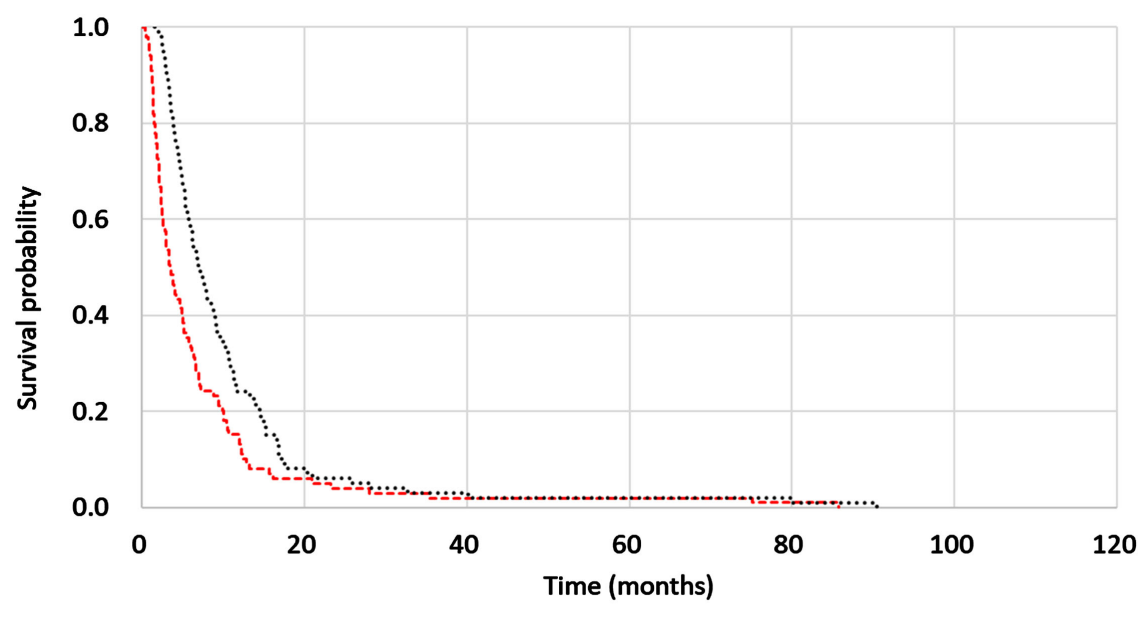

----- KM value to EHY …... KM HJP* value2

(a)

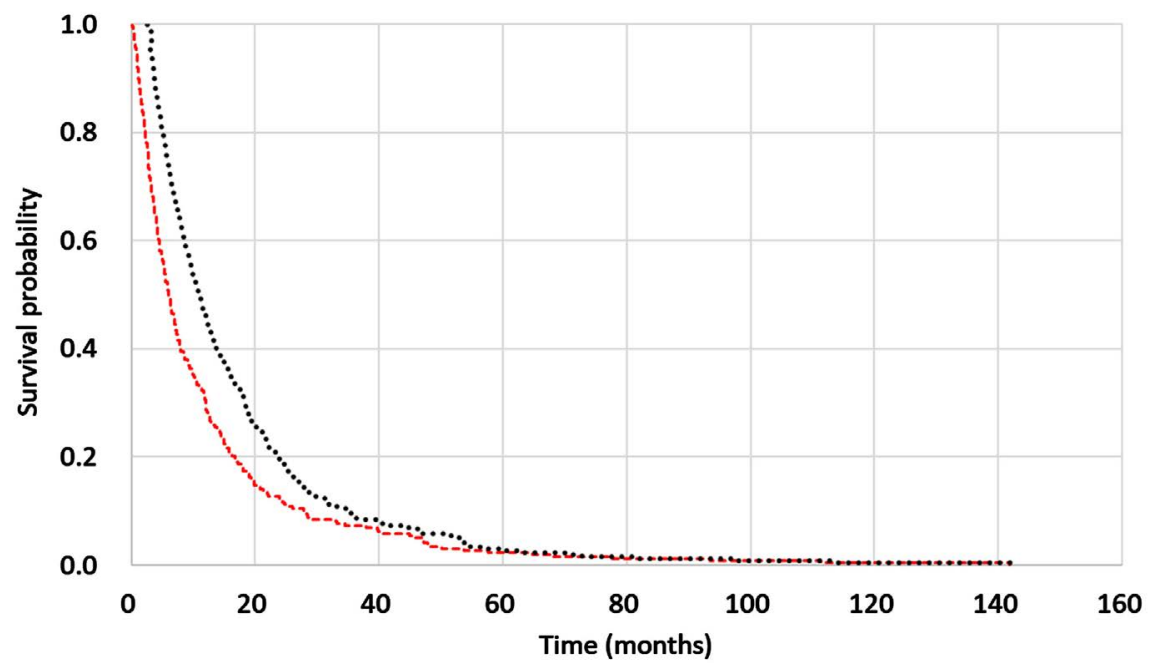

(b)

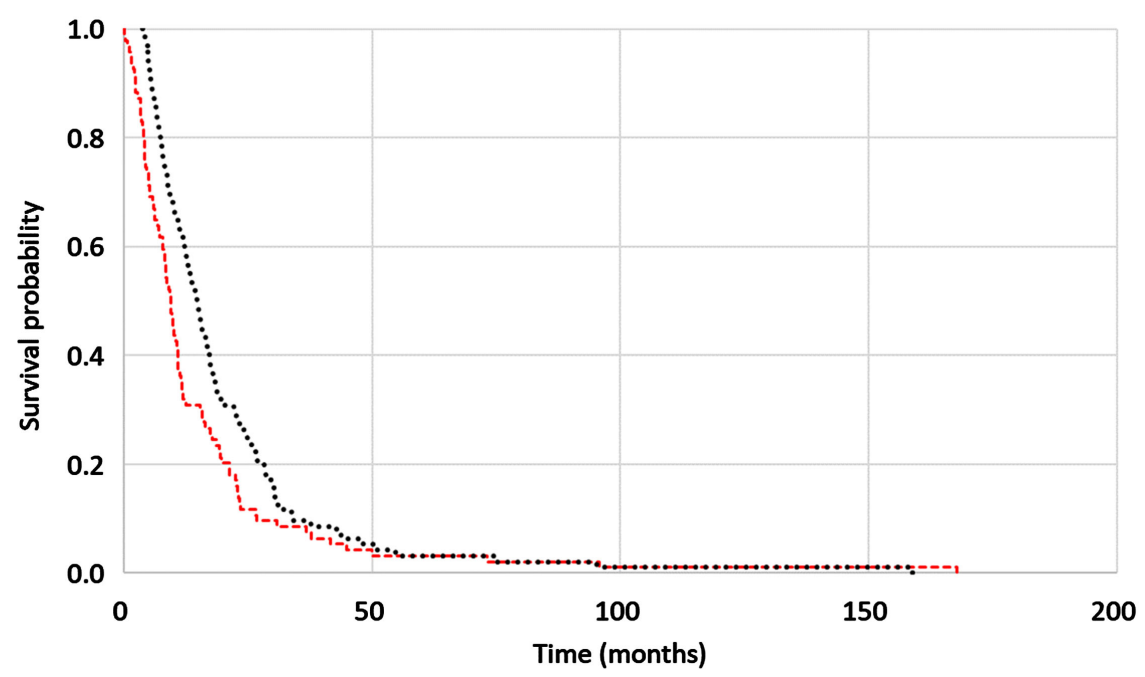

(c)

Figure 19. Comparison of HJPm curve (dotted line) and the elapsed time from the first diagnosis to the mEHT treatment (dashed line), (a) pancreas, (b) NSCLC, (c) GBM. 


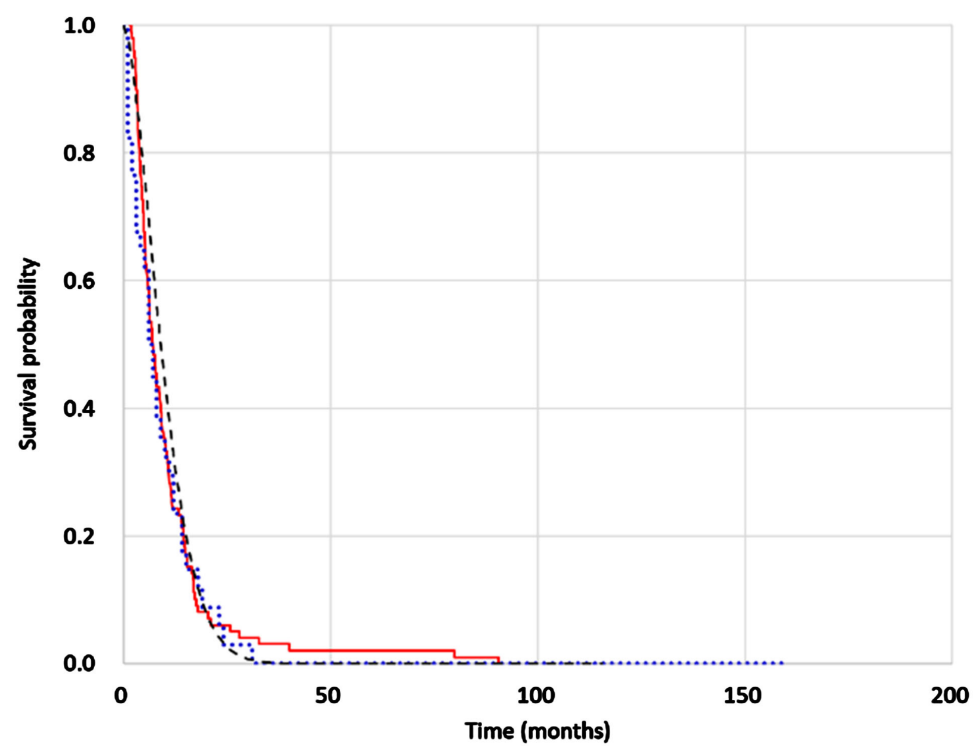

(a)

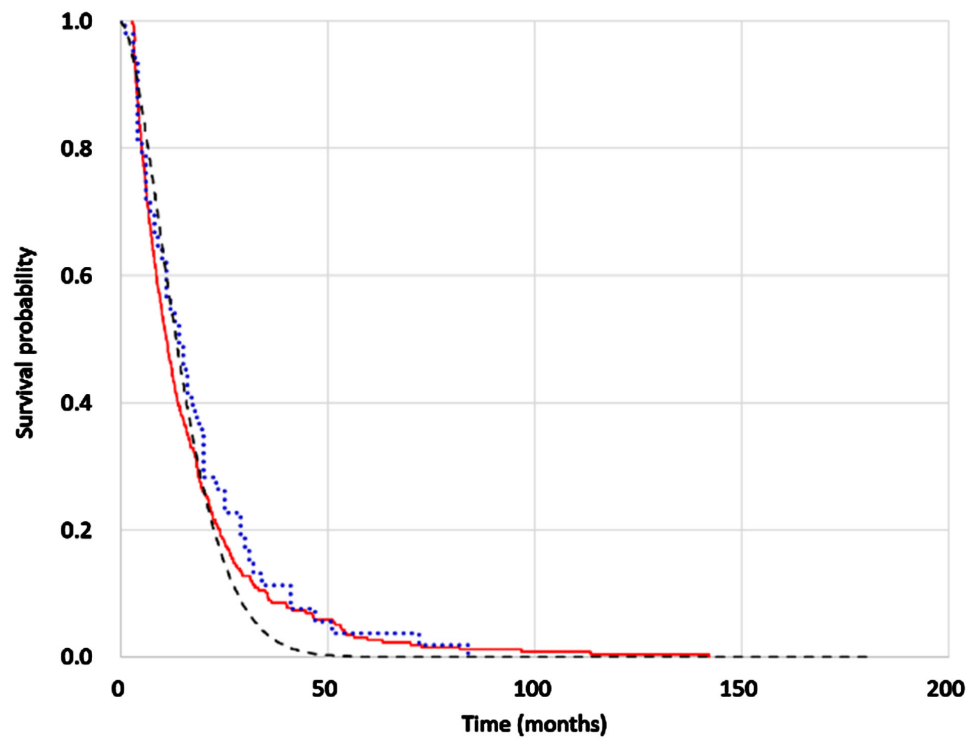

(b)

Figure 20. Comparison of the historical control (dotted line), the HJPm approximation (solid line) and the non-responding fraction from the WF decomposition (dashed line). (a) pancreas cancer, (65.6\% of weak conditions); (b) NSCLC (62.7\% of weak conditions).

subcomponents, so obtaining a single control arm is impossible, but the HJPm could be regarded as one of the possibilities. The relatively long time to the first mEHT treatment in the case of GBM creates longer expected survival too. Conventional curative possibilities take more time, and the patients start the mEHT mostly in terminal stages. In this case, $69.5 \%$ of the patients are grouped into the weak personal defensive conditions.

The curative benefit of mEHT complementary application is significant in comparison to the overall survival and the control-arm of the expected survival after the failure of conventional curative approaches (Figure 21). 


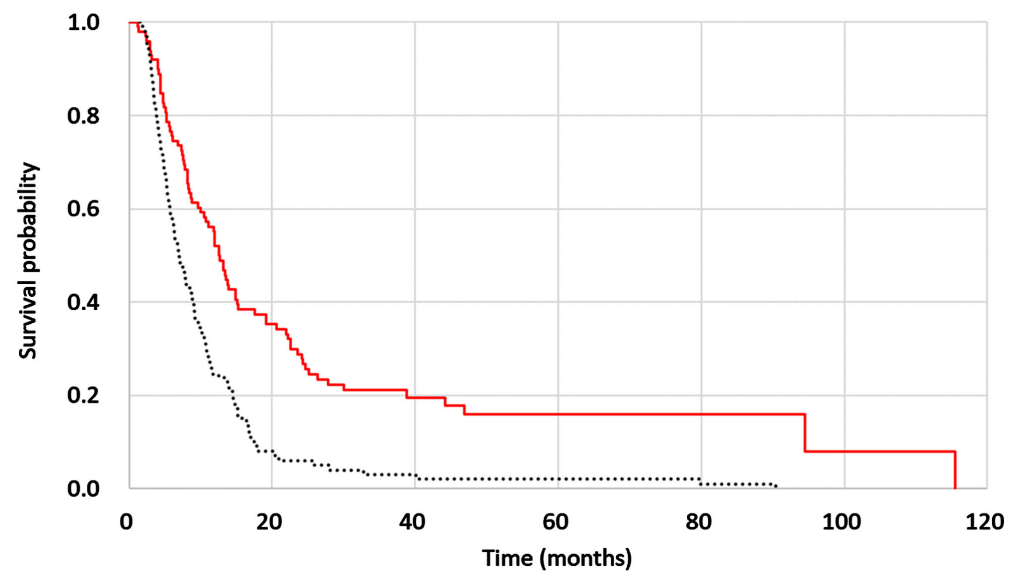

(a)

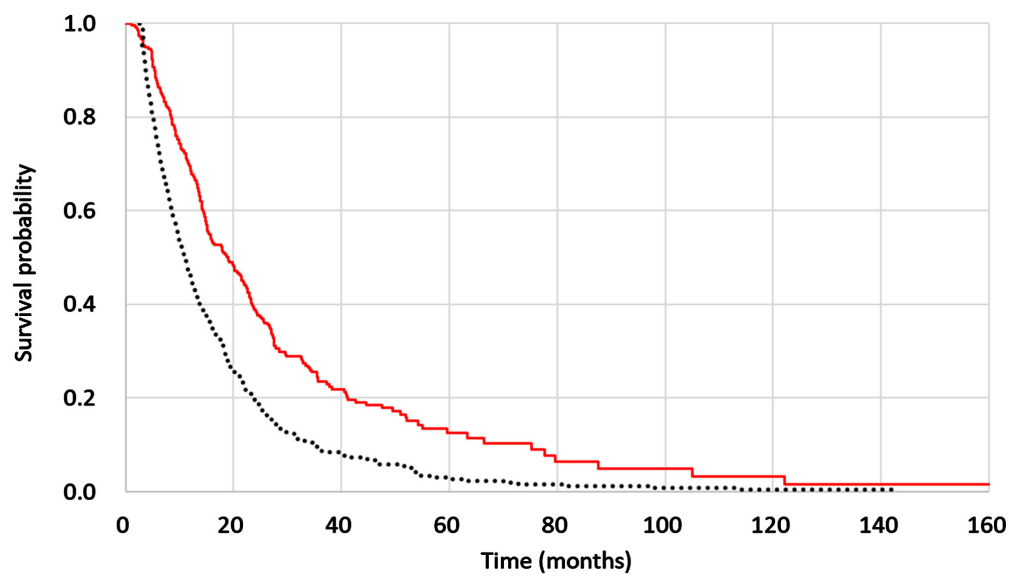

(b)

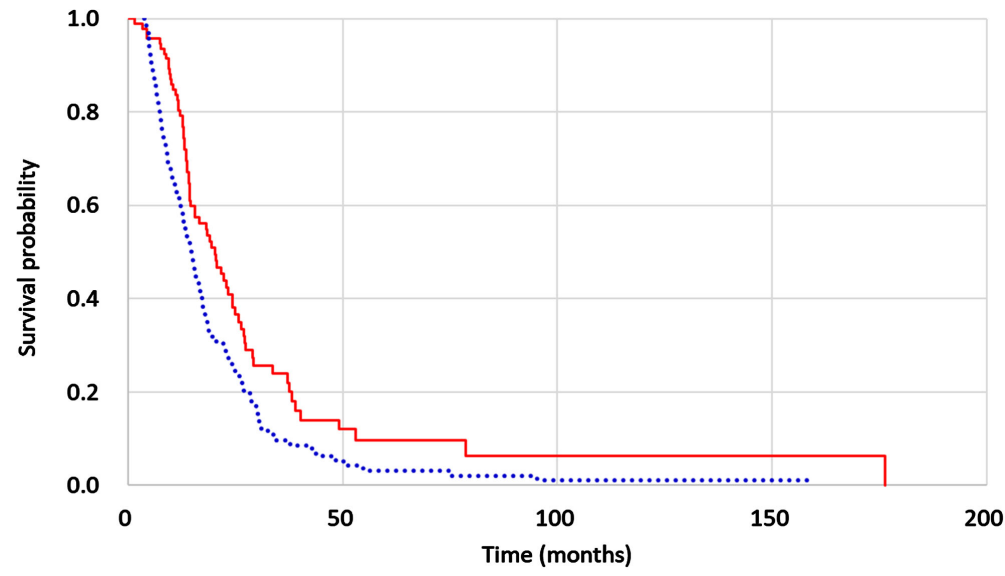

(c)

Figure 21. Comparison of the HJPm approximation as control (dotted line), with the overall survival (solid line). (a) pancreas cancer; (b) NSCLC; (c) GBM.

The research could be continued for a more complex single-arm research, where the QoL is taken into consideration too. The quality-adjusted survival (QAS) [78] [79], which considers the QAS without symptoms and toxicity (Q-TWIST) [80] would be an important extension to the single-arm study. 


\section{Conclusion}

The WF regression fit to KM non-parametric estimate works precisely in real clinical studies of advanced pancreatic cancer, NSCLC and GBM trials where the mEHT method was applied as a complementary treatment when no more conventional curative possibilities were available. The WF decomposition method creates an estimated reference-arm in a chosen homogeneous cohort. The control arm is correct, if we assume that patients start their mEHT treatment when the conventional therapies fail, so their overall status (relative to the lines of the conventional therapies) groups them into groups with similar conditions. The mEHT method has no harm for the patients (no adherent effects to make tumor-progress by the treatment alone), so the possibility of the treatment results has two categories only: effective or ineffective, which fits the decomposition concept well. Regression is accurate, and the control-arm from the decomposed WF corresponds well with the modified Hardin-Jones-Pauling statistical estimation too, when the number of patients is high enough $(>30)$ for statistical evaluation.

\section{Acknowledgements}

The research was supported by the Hungarian Competitiveness and Excellence Program grant (NVKP_16-1-2016-0042).

\section{Conflicts of Interest}

The authors declare no conflicts of interest regarding the publication of this paper.

\section{References}

[1] Szasz, O. and Szasz, A. (2018) Parameterization of Survival Measures (Part 1). International Journal of Clinical Medicine, 11, 316-347.

[2] Szasz, A., Szigeti, G.P. and Szasz, A.M. (2018) Parameterization of Survival Measures (Part 2). International Journal of Clinical Medicine, 11, 348-373.

[3] DeMets, D., Friedman, L. and Furberg, C. (2010) Fundamentals of Clinical Trials. 4th Edition, Springer, Berlin. https://doi.org/10.1007/978-1-4419-1586-3

[4] CDER (2006) "Exploratory IND Studies" Guidance for Industry, Investigators, and Reviewers. Food and Drug Administration, Silver Spring. https://link.springer.com/article/10.1177/009286150704100303

[5] Canadian Cancer Society (2017) Phases of Clinical Trials.

[6] Vernon, C.C., Hand, J.W., Field, S.B., Machin, D., Whaley, J.B., van der Zee, J., van Putten, W.L.J., van Rhoon, G.C., van Dijk, J.D.P., Gonzalez Gonzalez, D., Liu, F.-F., Goodman, P. and Sherar, M. (1996) Radiotherapy with or without Hyperthermia in the Treatment of Superficial Localized Breast Cancer: Results from Five Randomized Controlled Trials. International Journal of Radiation Oncology, Biology, Physics, 35, 731-744. https://doi.org/10.1016/0360-3016(96)00154-X

[7] Sherar, M., Liu, F.-F., Pintilie, M., et al. (1997) Relationship between Thermal Dose and Outcome in Thermoradiotherapy Treatments for Superficial Recurrences of Breast Cancer: Data from a Phase III Trial. International Journal of Radiation On- 
cology, Biology, Physics, 39, 371-380.

https://doi.org/10.1016/S0360-3016(97)00333-7

[8] Mitsumori, M., Zeng, Z.F., Oliynychenko, P., et al. (2007) Regional Hyperthermia Combined with Radiotherapy for Locally Advanced Non-Small Cell Lung Cancers. International Journal of Clinical Oncology, 12, 192-198. https://doi.org/10.1007/s10147-006-0647-5

[9] Shinn, K.S., Choi, I.B., Kay, C.S., et al. (1996) Thermoradiotherapy in the Treatment of Locally Advanced Nonsmall Cell Lung Cancer. The Journal of the Korean Society for Therapeutic Radiology and Oncology, 14, 115-122. https://doi.org/10.1016/0169-5002(96)85955-1

[10] Vasanthan, A., Mitsumori, M., Park, J.H., et al. (2005) Regional Hyperthermia Combined with Radiotherapy for Uterine Cervical Cancers: A Multi-Institutional Prospective Randomized Trial of the International Atomic Energy Agency. International Journal of Radiation Oncology, Biology, Physics, 61, 145-153. https://doi.org/10.1016/j.ijrobp.2004.04.057

[11] Zolciak-Siwinska, A., Piotrkowicz, N., Jonska-Gmyrek, J., et al. (2013) HDR Brachytherapy Combined with Interstitial Hyperthermia in Locally Advanced Cervical Cancer Patients Initially Treated with Concomitant Radiochemotherapy: A Phase III Study. Radiotherapy and Oncology, 109, 194-199. https://doi.org/10.1016/j.radonc.2013.04.011

[12] Jones, E.L., Oleson, J.R., Prosnitz, L.R., et al. (2005) Randomized Trial of Hyperthermia and Radiation for Superficial Tumors. Journal of Clinical Oncology, 23, 3079-3085. https://doi.org/10.1200/JCO.2005.05.520

[13] Evans, S.R. (2010) Clinical Trial Structures. Journal of Experimental Stroke \& Translational Medicine, 3, 8-18. https://doi.org/10.6030/1939-067X-3.1.8

[14] Arun, B., Austin, T., Babiera, G.V., et al. (2017) A Comprehensive Lifestyle Randomized Clinical Trial: Design and Initial Patient Experience. Integrative Cancer Therapies, 16, 3-20. https://doi.org/10.1177/1534735416679516

[15] Snyders, K., Cho, D., Hong, J.H., et al. (2019) Benchmarking Single-Arm Studies against Historical Controls from Non-Small Cell Lung Cancer Trials: An Empirical Analysis of Bias. Acta Oncologica, 59, 90-95.

https://doi.org/10.1080/0284186X.2019.1674452

[16] Rosenbaum, P.R. and Rubin, D.B. (1983) The Central Role of the Propensity Score in Observational Studies for Causal Effects. Biometrika, 70, 41-55. https://doi.org/10.1093/biomet/70.1.41

[17] Rosenbaum, P.R. and Rubin, D.B. (1984) Reducing Bias in Observational Studies Using Subclassification on the Propensity Score. Journal of the American Statistical Association, 79, 516-524. https://doi.org/10.1080/01621459.1984.10478078

[18] Rosenbaum, P.R. and Rubin, D.B. (1985) Constructing a Control Group Using Multivariate Matched Sampling Methods That Incorporate the Propensity Score. The American Statistician, 39, 33-38. https://doi.org/10.1080/00031305.1985.10479383

[19] Cochran, W.G. (1968) The Effectiveness of Adjustment by Sub-Classification in Removing Bias in Observational Studies. Biometrics, 24, 295-313. https://doi.org/10.2307/2528036

[20] Whitehead, J. (1999) A Unified Theory for Sequential Clinical Trials. Statistics in Medicine, 18, 2271-2286.

https://doi.org/10.1002/(SICI)1097-0258(19990915/30)18:17/18<2271::AID-SIM254 >3.0.CO;2-Z 
[21] Evans, C.H. and Ildstad, S.T. (2001) Small Clinical Trials: Issues and Challenges. National Academy Press, Washington DC. http://www.nap.edu/catalog/10078.html

[22] Bellissant, E., Benichou, J. and Chastang, C. (1996) The Group Sequential Triangular Test for Phase II Cancer Clinical Trials. American Journal of Clinical Oncology, 19, 422-430. https://doi.org/10.1097/00000421-199608000-00021

[23] O’Fallon, J.R. (1985) Policies for Interim Analysis and Interim Reporting of Results. Cancer Treatment Reports, 69, 1101-1116.

[24] Pocock, S.J. and Hughes, M.D. (1989) Practical Problems in Interim Analyses, with Particular Regard to Estimation. Controlled Clinical Trials, 10, 209S-221S. https://doi.org/10.1016/0197-2456(89)90059-7

[25] Emerson, S.S. and Fleming, T.R. (1990) Interim Analyses in Clinical Trials. Oncology, 4, 126-133.

[26] Whitehead, J. and Sooriyarachchi, M.R. (1998) A Method for Sequential Analysis of Survival Data with Non-Proportional Hazards. Biometrics, 54, 1072-1084. https://doi.org/10.2307/2533858

[27] Williams, P.L. (1996) Sequential Monitoring of Clinical Trials with Multiple Survival, Endpoints. Statistics in Medicine, 15, 2341-2357. https://doi.org/10.1002/(SICI)1097-0258(19961115)15:21<2341::AID-SIM453>3.0.C O;2-N

[28] Konishi, T., Watanabe, T., Kishimoto, J., et al. (2007) Prognosis and Risk Factors of Metastasis in Colorectal Carcinoids: Results of a Nationwide Registry over 15 Years. Gut, 56, 863-868. https://doi.org/10.1136/gut.2006.109157

[29] Weinber, R.A. (2007) The Biology of Cancer. Garland Science, New York. http://web.thu.edu.tw/cphu/www/cancer_biology/download/Ch_8_pRb_and_Contr ol_of_the_Cell_Cycle_Clock.pdf

[30] Roussakow, S. (2013) Critical Analysis of Electromagnetic Hyperthermia Randomized Trials: Dubious Effect and Multiple Biases. Conference Papers in Medicine, 2013, Article ID: 412186. https://doi.org/10.1155/2013/412186

[31] Roussakow, S. (2013) The History of Hyperthermia Rise and Decline. Conference Papers in Medicine, 2013, Article ID: 428027. https://doi.org/10.1155/2013/428027

[32] Szasz, A., Szasz, N. and Szasz, O. (2010) Oncothermia-Principles and Practices. Springer Science, Heidelberg. https://doi.org/10.1007/978-90-481-9498-8

[33] Howie, L. and Peppercorn, J. (2013) Early Palliative Care in Cancer Treatment: Rationale, Evidence and Clinical Implications. Therapeutic Advances in Medical Oncology, 5, 318-323. https://doi.org/10.1177/1758834013500375

[34] Ferrell, B.R., Temel, J.S., Temin, S., Alesi, E.R., Balboni, T.A., Basch, E.M., Firn, J.I., Paice, J.A., Peppercorn, J.M., Phillips, T., Stovall, E.L., Zimmermann, C. and Smith, T.J. (2016) Integration of Palliative Care into Standard Oncology Care: American Society of Clinical Oncology Clinical Practice Guideline Update. Journal of Clinical Oncology, 35, 96-112. https://doi.org/10.1200/JCO.2016.70.1474

[35] Isenberg, S.R., Aslakson, R.A. and Smith, T.J. (2017) Implementing Evidence-Based Palliative Care Programs and Policy for Cancer Patients: Epidemiologic and Policy Implications of the 2016 American Society of Clinical Oncology Clinical Practice Guideline Update. Epidemiologic Reviews, 39, 123-131. https://doi.org/10.1093/epirev/mxw002

[36] Andocs, G., Renner, H., Balogh, L., Fonyad, L., Jakab, C. and Szasz, A. (2009) Strong Synergy of Heat and Modulated Electro-Magnetic Field in Tumor Cell Killing, 
Study of HT29 Xenograft Tumors in a Nude Mice Model. Strahlentherapie und Onkologie, 185, 120-126. https://doi.org/10.1007/s00066-009-1903-1

[37] Hegyi, G., Szigeti, G.P. and Szasz, A. (2013) Hyperthermia versus Oncothermia: Cellular Effects in Complementary Cancer Therapy. Evidence-Based Complementary and Alternative Medicine, 2013, Article ID: 672873. https://doi.org/10.1155/2013/672873

[38] Meggyeshazi, N., Andocs, G., Balogh, L., Balla, P., Kiszner, G., Teleki, I., Jeney, A. and Krenacs, T. (2014) DNA Fragmentation and Caspase-Independent Programmed Cell Death by Modulated Electrohyperthermia. Strahlentherapie und Onkologie, 190, 815-822. https://doi.org/10.1007/s00066-014-0617-1

[39] Tsang, Y.-W., Huang, C.-C., Yang, K.-L., Chi, M.-S., Chiang, H.-C., Wang, Y.-S., Andocs, G., Szasz, A., Li, W.-T. and Chi, K.-H. (2015) Improving Immunological Tumor Microenvironment Using Electro-Hyperthermia Followed by Dendritic Cell Immunotherapy. BMC Cancer, 15, Article No. 708.

https://doi.org/10.1186/s12885-015-1690-2

[40] Qin, W., Akutsu, Y., Andocs, G., Sugnami, A., Hu, X., Yusup, G., Komatsu-Akimoto, A., Hoshino, I., Hanari, N., Mori, M., Isozaki, Y., Akanuma, N., Tamura, Y. and Matsubara, H. (2014) Modulated Electro-Hyperthermia Enhances Dendritic Cell Therapy through an Abscopal Effect in Mice. Oncology Reports, 32, 2373-2379. https://doi.org/10.3892/or.2014.3500

[41] Vancsik, T., Kovago, Cs., Kiss, E., Papp, E., Forika, G., Benyo, Z., Meggyeshazi, N. and Krenacs, T. (2018) Modulated Electro-Hyperthermia Induced Loco-Regional and Systemic Tumor Destruction in Colorectal Cancer Allografts. Journal of Cancer, 9, 41-53. https://doi.org/10.7150/jca.21520

[42] Andocs, G., Rehman, M.U., Zhao, Q.-L., Tabuchi, Y., Kanamori, M. and Kondo, T. (2016) Comparison of Biological Effects of Modulated Electro-Hyperthermia and Conventional Heat Treatment in Human Lymphoma U937 Cell. Cell Death Discovery (Nature Publishing Group), 2, 16039. https://doi.org/10.1038/cddiscovery.2016.39

[43] Yang, K.-L., Huang, C.-C., Chi, M.-S., Chiang, H.-C., Wang, Y.-S., Andocs, G., et al. (2016) In Vitro Comparison of Conventional Hyperthermia and Modulated Electro-Hyperthermia. Oncotarget, 7, 84082-84092. https://doi.org/10.18632/oncotarget.11444

[44] Szasz, A. (2013) Challenges and Solutions in Oncological Hyperthermia. Thermal Medicine, 29, 1-23. https://doi.org/10.3191/thermalmed.29.1

[45] Szasz, O. (2013) Renewing Oncological Hyperthermia-Oncothermia. Open Journal of Biophysics, 3, 245-252. https://doi.org/10.4236/ojbiphy.2013.34030

[46] Szasz, A. (2013) "Quo Vadis" Oncologic Hyperthermia? Conference Papers in Medicine, 2013, Article ID: 201671. https://doi.org/10.1155/2013/201671

[47] Szasz, A.M., Minnaar, C., Szentmartoni, Gy., Szigeti, G.P. and Dank, M. (2019) Review of the Clinical Evidences of Modulated Electro-Hyperthermia (mEHT) Method: An Update for the Practicing Oncologist. Frontiers in Oncology, 9, 1012. https://doi.org/10.3389/fonc.2019.01012

[48] Dani, A., Varkonyi, A., Magyar, T. and Szasz, A. (2008) Clinical Study for Advanced Pancreas Cancer Treated by Oncothermia. Forum Hyperthermie, 1, 13-20.

[49] Volovat, C., Volovat, S.R., Scripcaru, V., et al. (2014) Second-Line Chemotherapy with Gemcitabine and Oxaliplatin in Combination with Loco-Regional Hyperthermia (EHY-2000) in Patients with Refractory Metastatic Pancreatic Cancer: Preliminary Results of a Prospective Trial. Romanian Reports in Physics, 66, 166-174. 
[50] Douwes, F.R. (2006) Thermochemotherapy of the Advanced Pancreas Carcinoma. Biologische Medizin, 35, 126-130.

[51] Lee, D.Y., Haam, S.J., Kim, T.H., Lim, J.Y., Kim, E.J. and Kim, N.Y. (2013) Oncothermia with Chemotherapy in the Patients with Small Cell Lung Cancer. Conference Papers in Medicine, 2013, Article ID: 910363. https://doi.org/10.1155/2013/910363

[52] Dani, A., Varkonyi, A., Magyar, T. and Szasz, A. (2009) Clinical Study for Advanced Non-Small-Cell Lung-Cancer Treated by Oncothermia. Forum Hyperthermie, DGHT, Cologne, 26 September 2009.

[53] Szasz, A. (2014) Current Status of Oncothermia Therapy for Lung Cancer. The Korean Journal of Thoracic and Cardiovascular Surgery, 47, 77-93.

https://doi.org/10.5090/kjtcs.2014.47.2.77

[54] Ou, J., Zhu, X., Lu, Y., et al. (2017) The Safety and Pharmacokinetics of High Dose Intravenous Ascorbic Acid Synergy with Modulated Electrohyperthermia in Chinese Patients with Stage III-IV Non-Small Cell Lung Cancer. European Journal of Pharmaceutical Sciences, 109, 412-418. https://doi.org/10.1016/j.ejps.2017.08.011

[55] Sahinbas, H., Groenemeyer, D.H.W., Boecher, E. and Szasz, A. (2007) Retrospective Clinical Study of Adjuvant Electro-Hyperthermia Treatment for Advanced BrainGliomas. Deutsche Zeitschrift für Onkologie, 39, 154-160. https://doi.org/10.1055/s-2007-986020

[56] Wismeth, C., Dudel, C., Pascher, C., et al. (2010) Transcranial Electro-Hyperthermia Combined with Alkylating Chemotherapy in Patients with Relapsed High-Grade Gliomas-Phase I Clinical Results. Journal of Neuro-Oncology, 98, 395-405. https://doi.org/10.1007/s11060-009-0093-0

[57] Fiorentini, G., Giovanis, P., Rossi, S., et al. (2006) A Phase II Clinical Study on Relapsed Malignant Gliomas Treated with Electro-Hyperthermia. In Vivo, 20, 721-724.

[58] Roussakow, S. (2017) Clinical and Economic Evaluation of Modulated Electrohyperthermia Concurrent to Dose-Dense Temozolomide 21/28 Days Regimen in the Treatment of Recurrent Glioblastoma: A Retrospective Analysis of a Two-Centre German Cohort Trial with Systematic Comparison and Effect-to-Treatment Analysis, BMJ Open, 7, e017387. https://doi.org/10.1136/bmjopen-2017-017387

[59] Van Gool, S.W., Makalowski, J., Feyen, O., Prix, L., Schirrmacher, V. and Stuecker, W. (2018) The Induction of Immunogenic Cell Death (ICD) during Maintenance Chemotherapy and Subsequent Multimodal Immunotherapy for Glioblastoma (GBM). Austin Oncology Case Reports, 3, 1-8.

[60] Hager, E.D., Sahinbas, H., Groenemeyer, D.H., et al. (2008) Prospective Phase II Trial for Recurrent High-Grade Malignant Gliomas with Capacitive Coupled Low Radiofrequency (LRF) Deep Hyperthermia. Journal of Clinical Oncology, 26, 2047. https://doi.org/10.1200/jco.2008.26.15_suppl.2047

[61] Fiorentini, G., Sarti, D., Milandri, C., Dentioco, P., et al. (2018) Modulated Electrohyperthermia in Integrative Cancer Treatment for Relapsed Malignant Glioblastoma and Astrocytoma: Retrospective Multicenter Controlled Study. Integrative Cancer Therapies, 18, 1-11. https://doi.org/10.1177/1534735418812691

[62] Lee, S.-Y., Lee, N.-R., Cho, D.-H. and Kim, J.-S. (2017) Treatment Outcome Analysis of Chemotherapy Combined with Modulated Electro-Hyperthermia Compared with Chemotherapy Alone for Recurrent Cervical Cancer, Following Irradiation. Oncology Letters, 14, 73-78. https://doi.org/10.3892/ol.2017.6117

[63] Minnaar, C., Baeyens, A. and Kotzen, J. (2016) Update on Phase III Randomized Clinical Trial Investigating the Effects of the Addition of Electro-Hyperthermia to 
Chemoradiotherapy for Cervical Cancer Patients in South Africa. Physica Medica, 32, 151-152. https://doi.org/10.1016/j.ejmp.2016.07.042

[64] Ferrari, V.D., De Ponti, S., Valcamonico, F., et al. (2007) Deep Electro-Hyperthermia (EHY) with or without Thermo-Active Agents in Patients with Advanced Hepatic Cell Carcinoma: Phase II Study. Journal of Clinical Oncology, 25, 15168. https://doi.org/10.1200/jco.2007.25.18_suppl.15168

[65] Gadaleta-Caldarola, G., Infusino, S., Galise, I., et al. (2014) Sorafenib and Locoregional Deep Electro-Hyperthermia in Advanced Hepatocellular Carcinoma. A Phase II Study. Oncology Letters, 8, 1783-1787. https://doi.org/10.3892/ol.2014.2376

[66] Jeung, T.-S., Ma, S.-Y., Choi, J., et al. (2015) Results of Oncothermia Combined with Operation, Chemotherapy and Radiation Therapy for Primary, Recurrent and Metastatic Sarcoma. Case Reports in Clinical Medicine, 4, 157-168. https://doi.org/10.4236/crcm.2015.45033

[67] Volovat, C., Volovat, S.R., Scripcaru, V., et al. (2014) The Results of Combination of Ifosfamid and Locoregional Hyperthermia (EHY 2000) in Patients with Advanced Abdominal Soft-Tissue Sarcoma after Relapse of First Line Chemotherapy. Romanian Reports in Physics, 66, 175-181.

[68] Mambrini, A., Del Freo, A., Pacetti, P., et al. (2007) Intra-Arterial and Systemic Chemotherapy plus External Hyperthermia in Unresectable Biliary Cancer. Clinical Oncology, 19, 806-808. https://doi.org/10.1016/j.clon.2007.08.013

[69] Pang, C.L.K., Zhang, X., Wang, Z., Ou, J., Lu, Y., Chen, P., Zhao, C., et al. (2017) Local Modulated Electro-Hyperthermia in Combination with Traditional Chinese Medicine vs. Intraperitoneal Chemoinfusion for Treatment of Peritoneal Carcinomatosis with Malignant Ascites: A Phase II Randomized Trial. Molecular and Clinical Oncology, 6, 723-732. https://doi.org/10.3892/mco.2017.1221

[70] Hager, E.D. (2004) Lebermetastasen bei kolorektalen Karzinomen. Deutsche Zeitschrift für Onkologie, 36, 132-134.

[71] Hager, E.D., Dziambor, H., Höhmann, D., et al. (1999) Deep Hyperthermia with Radiofrequencies in Patients with Liver Metastases from Colorectal Cancer. Anticancer Research, 19, 3403-3408.

[72] Cover, T.M. and Thomas, J.A. (2005) Elements of Information Theory. Wiley, Hoboken. https://doi.org/10.1002/047174882X

[73] Pauling, L. (1989) Biostatistical Analysis of Mortality Data for Cohorts of Cancer Patients. Proceedings of the National Academy of Sciences of the United States of America, 86, 3466-3468. https://doi.org/10.1073/pnas.86.10.3466

[74] Herman, Z.S. (1998) On Understanding the Hardin Jones-Pauling Biostatistical Theory of Survival Analysis for Cancer Patients. The Journal of Orthomolecular Medicine, 13, 1-12.

[75] Herman, Z.S. (1998) The Application of the Hardin Jones-Pauling Biostatistical Theory of Survival Analysis for Cancer Patients to a Clinical Trial Purporting to Test the Efficacy of Vitamin C in Lengthening the Survival Times of Patients with Advanced Colorectal Cancer. The Journal of Orthomolecular Medicine, 13, 225-232.

[76] Pauling, L. and Herman, Z.S. (1989) Criteria for the Validity of Clinical Trials of Treatments of Cohorts of Cancer Patients Based on the Hardin Jones Principle. Proceedings of the National Academy of Sciences of the United States of America, 86, 6835-6837. https://doi.org/10.1073/pnas.86.18.6835

[77] Rouaud, M. (2013) Probability, Statistics and Estimation; Creative Commons Attribution-Non-Commercial 4.0 International License (CC BY-NC 4.0). 
[78] Glasziou, P.P., Simes, R.J. and Gelber, R.D. (1990) Quality Adjusted Survival Analysis. Statistics in Medicine, 9, 1259-1276. https://doi.org/10.1002/sim.4780091106

[79] Martin, A.J. and Simes, R.J. (2013) Quality-Adjusted Survival as an End Point in Breast Cancer Trials. Journal of Clinical Investigation, 3, 545-555. https://doi.org/10.4155/cli.13.37

[80] Cole, B.F., Gelber, R.D., Gelber, S. and Mukhopadhyay, P. (2014) A Quality-Adjusted Survival (Q-TWiST) Model for Evaluating Treatments for Advanced Stage Cancer. Journal of Biopharmaceutical Statistics, 14, 111-124.

https://doi.org/10.1081/BIP-120028509 